\title{
Stereoselective total synthesis of sporiolide B and attempted synthesis of sporiolide $A$
}

\author{
Gattu Sridhar* and Gangavaram V. M. Sharma \\ Organic and Biomolecular Chemistry Division, \\ CSIR-Indian Institute of Chemical Technology, Hyderabad, 500107, India. \\ (Email: sridhar1org@yahoo.com) \\ SUPPORTING INFORMATION \\ CONTENTS
}

General information

S2

Experimental section

S2-S17

Copies of ${ }^{1} \mathrm{H}$ and ${ }^{13} \mathrm{C}$ NMR spectra of all new compounds

S18-S45 


\section{GENERAL PROCEDURES}

Solvents were dried over standard drying agents and freshly distilled prior to use. All commercially available chemicals were used without further purification. All reactions were performed under Nitrogen. ${ }^{1} \mathrm{H}$ NMR and ${ }^{13} \mathrm{C}$ NMR spectra were measured with Varian Gemini FT 200 MHz spectrometer, Bruker Avance 300 MHz, Unity $400 \mathrm{MHz}$ and Inova 500 $\mathrm{MHz}$ with tetramethylsilane as internal standard for solutions in $\mathrm{CDCl}_{3} . J$ values are given in Hz. Chemical shifts were reported in $\mathrm{ppm}$ relative to solvent signal. All column chromatographic separations were performed using silica gel (Acme's, 60-120 mesh). Organic solutions were dried over anhydrous $\mathrm{Na}_{2} \mathrm{SO}_{4}$ and concentrated below $40{ }^{\circ} \mathrm{C}$ in vacuo. IR-spectra were recorded on FT IR (Perkin-Elmer IR-683) spectrophotometer with $\mathrm{NaCl}$ optics. JASCO DIP 300 digital polarimeter was used for measurement of optical rotations at

$25{ }^{\circ} \mathrm{C}$. Mass spectra were recorded on direct inlet system or LC by MSD trap SL (Agilent Technologies), the HRMS data were obtained using Q-TOF mass spectrometry.

\section{(R)-(2-(Benzyloxy)-3-(4-methoxybenzyloxy)propoxy)(tert.-butyl)dimethylsilane (14):}

To an ice cooled suspension of $\mathrm{NaH}(3.53 \mathrm{~g}, 147.23 \mathrm{mmol})$ in THF (120 mL), alcohol 12 (12 g, $36.80 \mathrm{mmol}$ ) was added, stirred for $30 \mathrm{~min}$ and treated with a solution of $\mathrm{BnBr}(5.25$ $\mathrm{mL}, 44.17 \mathrm{mmol})$ in THF $(30 \mathrm{~mL})$ and stirred at $35{ }^{\circ} \mathrm{C}$ temperature for $6 \mathrm{~h}$. The reaction mixture was quenched with sat. $\mathrm{NH}_{4} \mathrm{Cl}$ solution $(20 \mathrm{~mL})$ and extracted with ethyl acetate $(2 \mathrm{x}$ $100 \mathrm{~mL})$. The organic layers were washed with water $(2 \times 50 \mathrm{~mL})$, brine $(100 \mathrm{~mL})$ and dried $\left(\mathrm{Na}_{2} \mathrm{SO}_{4}\right)$. Solvent was evaporated under reduced pressure and purified the residue by column chromatography (60-120 mesh silica gel, 8\% ethyl acetate in pet. ether) to furnish 14 (13.1 g, $85 \%$ ) as a yellow liquid with $100 \%$ diastereoselectivity. $[\alpha]^{25}=+4.8\left(c 0.5, \mathrm{CHCl}_{3}\right)$; IR (neat): $3018,2930,2857,1612,1513,1466,1249,1214,1175,1094,1035,928,837,743$, $667 \mathrm{~cm}^{-1} ;{ }^{1} \mathrm{H}$ NMR $\left(\mathrm{CDCl}_{3}, 300 \mathrm{MHz}\right): \delta$ 7.35-7.27 (m, 5H, ArH-Bn), 7.22 (d, 2H, $J=8.6$ Hz, ArH-PMB), 6.83 (d, 2H, $J=8.6 \mathrm{~Hz}$, ArH-PMB), 4.65 (s, 2H, benzylic), 4.44 (s, 2H, 
benzylic), $3.75\left(\mathrm{~s}, 3 \mathrm{H},-\mathrm{OCH}_{3}\right), 3.68\left(\mathrm{~d}, 2 \mathrm{H}, \mathrm{J}=4.8 \mathrm{~Hz},-\mathrm{OCH}_{2}\right), 3.64-3.55\left(\mathrm{~m}, 2 \mathrm{H},-\mathrm{OCH}_{2}\right)$, 3.52-3.48 (m, 1H, -OCH), $0.90\left(\mathrm{~s}, 9 \mathrm{H}, 3 \mathrm{x}-\mathrm{CH}_{3}\right), 0.06\left(\mathrm{~s}, 6 \mathrm{H}, 2 \mathrm{x}-\mathrm{CH}_{3}\right) ;{ }^{13} \mathrm{C} \mathrm{NMR}\left(\mathrm{CDCl}_{3}\right.$, $75 \mathrm{MHz}): \delta 158.9,138.7,129.0,128.0,127.4,127.2,113.5,78.7,72.8,72.0,69.6,62.9,54.9$, 25.7, 18.1, -5.4; HRMS (ESI): $\mathrm{m} / \mathrm{z}$ calculated for $\mathrm{C}_{24} \mathrm{H}_{40} \mathrm{O}_{4} \mathrm{NSi}\left[\mathrm{M}+\mathrm{NH}_{4}\right]^{+} 434.2714$, found 434.2721 .

\section{(S)-2-(Benzyloxy)-3-(4-methoxybenzyloxy)propan-1-ol (15):}

To a solution of $\mathbf{1 4}(11.5 \mathrm{~g}, 27.64 \mathrm{mmol})$ in THF $(115 \mathrm{~mL})$ at $0{ }^{\circ} \mathrm{C}, 1 \mathrm{M}$ TBAF in THF $(33 \mathrm{~mL})$ was added and stirred for $1 \mathrm{~h}$. It was diluted with water $(50 \mathrm{~mL})$ and extracted with EtOAc $(2 \times 50 \mathrm{~mL})$. The combined organic layers were washed with water $(2 \times 50 \mathrm{~mL})$, brine $(50 \mathrm{~mL})$, dried $\left(\mathrm{Na}_{2} \mathrm{SO}_{4}\right)$, evaporated and purified the residue by column chromatography (60-120 mesh silica gel, 13\% ethyl acetate in pet. ether) to give $15(7.1 \mathrm{~g}, 85 \%)$ as a yellow liquid with $100 \%$ diastereoselectivity. $[\alpha]_{\mathrm{D}}^{25}=+28.3$ (c 0.5, $\mathrm{CHCl}_{3}$ ); IR (neat): 3447, 2930, $1719,1636,1454,1268,1217,1102,1035,916,771 \mathrm{~cm}^{-1} ;{ }^{1} \mathrm{H} \mathrm{NMR}\left(\mathrm{CDCl}_{3}, 300 \mathrm{MHz}\right): \delta$ 7.35-7.28 (m, 5H, ArH-Bn), 7.24 (d, 2H, $J=8.5 \mathrm{~Hz}$, ArH-PMB), 6.87 (d, 2H, $J=8.6 \mathrm{~Hz}$, ArH-PMB), 4.65 (dd, 2H, $J=11.7,46.0 \mathrm{~Hz}$, benzylic), $4.46(\mathrm{~d}, J=11.7 \mathrm{~Hz}, 2 \mathrm{H}$, benzylic), $3.80\left(\mathrm{~s}, 3 \mathrm{H},-\mathrm{OCH}_{3}\right), 3.76-3.72(\mathrm{~m}, 1 \mathrm{H},-\mathrm{OCH}), 3.69-3.64(\mathrm{~m}, 2 \mathrm{H}, 2 \mathrm{x}-\mathrm{OCH}), 3.62-3.55(\mathrm{~m}$, $\left.2 \mathrm{H},-\mathrm{OCH}_{2}\right) ;{ }^{13} \mathrm{C} \mathrm{NMR}\left(\mathrm{CDCl}_{3}, 75 \mathrm{MHz}\right): \delta 159.1,138.2,128.4,128.2,127.7,127.5,126.3$, 117.4, 96.1, 81.0, 79.3, 73.5, 60.4, 55.8; HRMS (ESI): $\mathrm{m} / \mathrm{z}$ calculated for $\mathrm{C}_{18} \mathrm{H}_{22} \mathrm{O}_{4} \mathrm{Na}$ $[\mathrm{M}+\mathrm{Na}]^{+}$325.1402, found 325.1409.

\section{(S,E)-Ethyl 4-(benzyloxy)-5-(4-methoxybenzyloxy)pent-2-enoate (16):}

To a solution of oxalyl chloride $(2.98 \mathrm{~mL}, 34.72 \mathrm{mmol})$ in dry $\mathrm{CH}_{2} \mathrm{Cl}_{2}(30 \mathrm{~mL})$ at -78 ${ }^{\circ} \mathrm{C}$, dry DMSO $(9.48 \mathrm{~mL}, 69.53 \mathrm{mmol})$ was added dropwise and stirred for $20 \mathrm{~min}$. A solution of $15(7 \mathrm{~g}, 23.17 \mathrm{mmol})$ in dry $\mathrm{CH}_{2} \mathrm{Cl}_{2}(70 \mathrm{~mL})$ was added and stirred for $2 \mathrm{~h}$ at -78

${ }^{\circ} \mathrm{C}$. It was quenched with $\mathrm{Et}_{3} \mathrm{~N}(19.34 \mathrm{~mL}, 139.0 \mathrm{mmol})$ and diluted with $\mathrm{CH}_{2} \mathrm{Cl}_{2}(80 \mathrm{~mL})$. 
The reaction mixture was washed with water $(80 \mathrm{~mL})$, brine $(38 \mathrm{~mL})$, dried $\left(\mathrm{Na}_{2} \mathrm{SO}_{4}\right)$ and evaporated to furnish the corresponding aldehyde $\mathbf{1 5 a}$.

Aldehyde 15a (7 g, $23.33 \mathrm{mmol})$ was dissolved in benzene $(70 \mathrm{~mL})$ and treated with (ethoxycarbonylmethylene)triphenyl phosphorane $(11.69 \mathrm{~g}, 35.0 \mathrm{mmol})$ at reflux for $2 \mathrm{~h}$. Solvent was evaporated and purified the residue by column chromatography (60-120 mesh silica gel, 10\% ethyl acetate in pet. ether) to furnish $16(7.5 \mathrm{~g}, 87 \%)$ as a yellow liquid. The diasterioselectivity of $\mathrm{E}$ and $\mathrm{Z}$ isomers are 98:2. $[\alpha]_{\mathrm{D}}^{25}=+63.8\left(\right.$ c $\left.0.5, \mathrm{CHCl}_{3}\right)$; IR (neat): $3063,3031,2979,2923,2856,1716,1657,1611,1586,1512,1455,1390,1366,1346,1246$, 1174, 1092, 1033, 982, 820, 737, $698 \mathrm{~cm}^{-1} ;{ }^{1} \mathrm{H}$ NMR $\left(\mathrm{CDCl}_{3}, 300 \mathrm{MHz}\right): \delta$ 7.36-7.23 (m, 7H, ArH-Bn, ArH-PMB), 6.94-6.86 (m, 3H, ArH-PMB, olefinic), 6.11 (d, 1H, $J=15.86 \mathrm{~Hz}$, olefinic), 4.67-4.49 (m, 4H, benzylic), 4.25-4.17 (m, 3H, - $\left.\mathrm{OCH}_{2},-\mathrm{OCH}\right), 3.80(\mathrm{~s}, 3 \mathrm{H}$, $\left.\mathrm{OCH}_{3}\right), 3.62-3.50\left(\mathrm{~m}, 2 \mathrm{H},-\mathrm{OCH}_{2}\right), 1.30\left(\mathrm{t}, 3 \mathrm{H}, J=7.17 \mathrm{~Hz},-\mathrm{CH}_{3}\right) ;{ }^{13} \mathrm{C} \mathrm{NMR}\left(\mathrm{CDCl}_{3}, 75\right.$ MHz): $\delta 166.1,159.2,145.2,137.9,129.9,129.3,128.3,127.6,123.0,113.7,73.1,71.8,71.5$, 60.5, 55.2, 14.2; HRMS (ESI): $\mathrm{m} / \mathrm{z}$ calculated for $\mathrm{C}_{22} \mathrm{H}_{30} \mathrm{O}_{5} \mathrm{~N}\left[\mathrm{M}+\mathrm{NH}_{4}\right]^{+} 388.2120$, found 388.2118 .

\section{(S,E)-4-(Benzyloxy)-5-(4-methoxybenzyloxy)pent-2-en-1-ol (17):}

To a stirred solution of $\mathbf{1 6}(7.3 \mathrm{~g}, 19.73 \mathrm{mmol})$ in dry $\mathrm{CH}_{2} \mathrm{Cl}_{2}(70 \mathrm{~mL})$, DIBAL-H (22.4 mL, $39.43 \mathrm{mmol}, 20 \%$ solution in hexane) was added at $0{ }^{\circ} \mathrm{C}$ and for $2 \mathrm{~h}$. Methanol (30

$\mathrm{mL}$ ) was added to the reaction mixture at this temperature and stirred for $10 \mathrm{~min}$. The reaction mixture was diluted with EtOAc $(50 \mathrm{~mL})$, aq. potassium sodium tartrate $(10 \mathrm{~mL})$ and stirred vigorously at $35^{\circ} \mathrm{C}$ temperature for an additional $1 \mathrm{~h}$. It was filterd through celite and the filtrate dried $\left(\mathrm{Na}_{2} \mathrm{SO}_{4}\right)$. Solvent was evaporated and the residue purified by column chromatography (60-120 mesh silica gel, 13\% ethyl acetate in pet. ether) to furnish $\mathbf{1 7}$ (5.6 g, $86 \%$ ) as colorless syrup with $100 \%$ diastereoselectivity. $[\alpha]^{25}{ }_{D}=+119.3\left(c 0.5, \mathrm{CHCl}_{3}\right)$; IR 
(neat): $3453,2860,1636,1513,1456,1248,1217,1175,1093,1033,760,671 \mathrm{~cm}^{-1} ;{ }^{1} \mathrm{H}$ NMR (300 MHz, $\left.\mathrm{CDCl}_{3}\right): \delta$ 7.37-7.29 (m, 5H, ArH-Bn), 7.25 (d, 2H, J=8.93 Hz, ArH-PMB), 6.86 (d, 2H, $J=8.69 \mathrm{~Hz}$, ArH-PMB), 5.93-5.88 (m, 1H, olefinic), 5.70-5.64 (m, 1H, olefinic), 4.64-4.46 (m, 4H, benzylic), $4.16\left(\mathrm{~d}, 2 \mathrm{H}, J=5.34 \mathrm{~Hz},-\mathrm{OCH}_{2}\right), 4.08-4.04(\mathrm{~m}, 1 \mathrm{H},-\mathrm{OCH})$, $3.80\left(\mathrm{~s}, 3 \mathrm{H},-\mathrm{OCH}_{3}\right), 3.58-3.49\left(\mathrm{~m}, 2 \mathrm{H},-\mathrm{OCH}_{2}\right) ;{ }^{13} \mathrm{C} \mathrm{NMR}\left(\mathrm{CDCl}_{3}, 75 \mathrm{MHz}\right): \delta 159.1,138.4$, $133.1,130.2,129.2,128.7,128.3,127.6,127.4,113.7,78.3,72.9,72.6,70.6,62.8,55.2$; HRMS (ESI): $m / z$ calculated for $\mathrm{C}_{20} \mathrm{H}_{28} \mathrm{NO}_{4}\left[\mathrm{M}+\mathrm{NH}_{4}\right]^{+}$346.2009, found 346.2012.

\section{((2S,3R)-3-((R)-1-(Benzyloxy)-2-(4-methoxybenzyloxy)ethyl)oxiran-2-yl)methanol (18):}

To a stirred solution of (+)-DIPT $(0.78 \mathrm{~g}, 3.35 \mathrm{mmol})$ in $\mathrm{CH}_{2} \mathrm{Cl}_{2}(30 \mathrm{~mL})$ at $-20{ }^{\circ} \mathrm{C}$ containing MS $4 \AA(3 \mathrm{~g}), \operatorname{Ti}\left(\mathrm{O}^{i} \mathrm{Pr}\right)_{4}(0.49 \mathrm{~mL}, 1.67 \mathrm{mmol})$ and cumene hydroperoxide $(3.76$ $\mathrm{mL}, 25.15 \mathrm{mmol}$ ) were added sequentially and stirred for $20 \mathrm{~min}$. A solution of allylic alcohol 17 (5.5 g, $16.76 \mathrm{mmol})$ in $\mathrm{CH}_{2} \mathrm{Cl}_{2}(30 \mathrm{~mL})$ was added and stirred at $-20{ }^{\circ} \mathrm{C}$ for $5 \mathrm{~h}$. The reaction mixture was quenched with $10 \% \mathrm{NaOH}$ solution $(5.5 \mathrm{~g}$ in $55 \mathrm{~mL}$ brine $)$ and stirred for $3 \mathrm{~h}$. It was filtered through a pad of celite and washed with $\mathrm{CH}_{2} \mathrm{Cl}_{2}(60 \mathrm{~mL})$. The organic layer was dried $\left(\mathrm{Na}_{2} \mathrm{SO}_{4}\right)$, evaporated and purified the residue by column chromatography (60-120 mesh silica gel, $15 \%$ ethyl acetate in pet. ether) to furnish $\mathbf{1 8}$ (4.9 $\mathrm{g}, 85 \%)$ as a yellow oil with $100 \%$ diastereoselectivity. $[\alpha]^{25}=+107.1$ (c $\left.0.5, \mathrm{CHCl}_{3}\right)$; IR (neat): 3447 , $2908,2865,1720,1611,1585,1513,1457,1364,1301,1248,1093,1032,899,699 \mathrm{~cm}^{-1} ;{ }^{1} \mathrm{H}$ NMR (300 MHz, $\left.\mathrm{CDCl}_{3}\right): \delta$ 7.37-7.27 (m, 5H, ArH-Bn), $7.23(\mathrm{~d}, 2 \mathrm{H}, J=8.69 \mathrm{~Hz}, \mathrm{ArH}-$ PMB), 6.87 (d, 2H, $J=8.54 \mathrm{~Hz}$, ArH-PMB), 4.79-4.61 (m, 2H, benzylic), 4.50-4.43 (m, 2H, benzylic), 3.94-3.83 (m, 1H, -OCH), $3.80\left(\mathrm{~s}, 3 \mathrm{H},-\mathrm{OCH}_{3}\right), 3.65-3.53(\mathrm{~m}, 3 \mathrm{H}, 3$ x $-\mathrm{OCH})$, 3.43-3.40 (m, 1H, -OCH), 3.18-3.02 (m, 2H, epoxy), 3.58-3.49 (m, 2H, $\left.-\mathrm{OCH}_{2}\right) ;{ }^{13} \mathrm{C}$ NMR $\left(\mathrm{CDCl}_{3}, 75 \mathrm{MHz}\right): \delta 159.1,138.0,129.8,129.2,128.3,127.7,127.6,113.7,78.0,73.1,72.0$, 69.8, 61.2, 56.6, 55.2; HRMS (ESI): $\mathrm{m} / \mathrm{z}$ calculated for $\mathrm{C}_{20} \mathrm{H}_{28} \mathrm{NO}_{5}\left[\mathrm{M}+\mathrm{NH}_{4}\right]^{+} 362.1963$, found 362.1969 . 
(3R,4R)-4-(Benzyloxy)-5-(4-methoxybenzyloxy)pentane-1,3-diol (19):

To a stirred solution of epoxide 18 (4.8 g, $13.95 \mathrm{mmol})$ in dry THF (50 mL), Red-Al $\left(5.5 \mathrm{~mL}, 27.87 \mathrm{mmol}, 70 \% \mathrm{w} / \mathrm{w}\right.$ in toluene) was added at $0{ }^{\circ} \mathrm{C}$. After $4 \mathrm{~h}$, the reaction mixture was quenched with sat. $\mathrm{NH}_{4} \mathrm{Cl}$ solution $(15 \mathrm{~mL})$ and filtered through a pad of celite and washed with EtOAc $(2 \times 100 \mathrm{~mL})$. Organic layers were dried $\left(\mathrm{Na}_{2} \mathrm{SO}_{4}\right)$, evaporated solvent and purified the residue by column chromatography (60-120 mesh silica gel, 25\% ethyl acetate in pet. ether) to afford $19(4.1 \mathrm{~g}, 85 \%)$ as a colorless oil with $100 \%$ diastereoselectivity. $[\alpha]_{\mathrm{D}}^{25}=+119.4\left(\right.$ c 0.5, $\left.\mathrm{CHCl}_{3}\right)$; IR (neat): 3401, 2984, 2940, 2878, 1715, 1651, 1419, 1373, 1217, 1159, 1055, $862 \mathrm{~cm}^{-1} ;{ }^{1} \mathrm{H}$ NMR (300 MHz, $\left.\mathrm{CDCl}_{3}\right): \delta$ 7.36-7.29 (m, 5H, ArH-Bn), 7.25 (d, 2H, $J=8.69 \mathrm{~Hz}, \mathrm{ArH}-\mathrm{PMB}), 6.88$ (d, 2H, $J=8.54 \mathrm{~Hz}, \mathrm{ArH}-\mathrm{PMB})$, 4.72 (dd, 1H, $J=11.59,23.95 \mathrm{~Hz}$, benzylic), 4.59-4.45 (m, 3H, benzylic), 3.99 (m, 1H, $\mathrm{OCH}), 3.81\left(\mathrm{~s}, 3 \mathrm{H},-\mathrm{OCH}_{3}\right), 3.80(\mathrm{~m}, 1 \mathrm{H},-\mathrm{OCH}), 3.71-3.65(\mathrm{~m}, 2 \mathrm{H}, 2 \mathrm{x}-\mathrm{OCH}), 3.60-3.48$ (m, 2H, 2 x -OCH) $1.82-1.65\left(\mathrm{~m}, 2 \mathrm{H},-\mathrm{CH}_{2}\right) ;{ }^{13} \mathrm{C} \mathrm{NMR}\left(\mathrm{CDCl}_{3}, 75 \mathrm{MHz}\right): \delta 163.4,142.4$, $133.6,132.5,132.1,132.0,131.9,118.0,84.7,81.4,74.9,73.8,64.6,59.4,39.1$; HRMS (ESI): $m / z$ calculated for $\mathrm{C}_{20} \mathrm{H}_{26} \mathrm{O}_{5} \mathrm{Na}[\mathrm{M}+\mathrm{Na}]^{+} 369.1669$, found 369.1672 .

(2R,3R)-2-(Benzyloxy)-5-(tert.-butyldimethylsilyloxy)-1-(4-methoxybenzyloxy)pentan-3ol (11):

To a cooled $\left(0{ }^{\circ} \mathrm{C}\right)$ solution of $19(4 \mathrm{~g}, 11.56 \mathrm{mmol})$ in $\mathrm{CH}_{2} \mathrm{Cl}_{2}(40 \mathrm{~mL})$, imidazole (2.35 g, $34.68 \mathrm{mmol})$, dibutyltin oxide $(0.005 \mathrm{~g}, 0.02 \mathrm{mmol})$ was added. After $30 \mathrm{~min}$ TBDMS-Cl $(1.73 \mathrm{~g}, 11.56 \mathrm{mmol})$ was added and stirred at $35{ }^{\circ} \mathrm{C}$ temperature for $2 \mathrm{~h}$. The reaction mixture was evaporated and purified the residue by column chromatography (60-120 mesh silica gel, 11\% EtOAc in pet. ether) to afford 11 (4.8 g, 90\%) as a colorless liquid with $100 \%$ diastereoselectivity. $[\alpha]^{25}=+98.4\left(c\right.$ 0.5, $\left.\mathrm{CHCl}_{3}\right)$; IR (neat): 3455, 2953, 2930, 2857, $1718,1610,1513,1464,1390,1251,1092,1034,773 \mathrm{~cm}^{-1} ;{ }^{1} \mathrm{H} \mathrm{NMR}\left(\mathrm{CDCl}_{3}, 300 \mathrm{MHz}\right): \delta$ 7.34-7.24 (m, 7H, ArH-Bn, ArH-PMB), 6.87 (d, 2H, J = 8.54 Hz, ArH-PMB), 4.75 (dd, 1H, $J$ 
$=9.3,11.59 \mathrm{~Hz}$, benzylic), $4.59(\mathrm{t}, 1 \mathrm{H}, J=11.59 \mathrm{~Hz}$, benzylic), 4.51-4.44 (m, 2H, benzylic), 3.97-3.83 (m, 2H, $\left.-\mathrm{OCH}_{2}\right), 3.80\left(\mathrm{~s}, 3 \mathrm{H},-\mathrm{OCH}_{3}\right), 3.77-3.61\left(\mathrm{~m}, 3 \mathrm{H},-\mathrm{OCH}_{2},-\mathrm{OCH}\right), 3.54-3.51$ (m, 1H, -OCH), 1.87-1.65 (m, 2H, $\left.-\mathrm{CH}_{2}\right), 0.89\left(\mathrm{~s}, 9 \mathrm{H}, 3 \mathrm{x}-\mathrm{CH}_{3}\right), 0.06(\mathrm{~d}, 6 \mathrm{H}, J=4.12 \mathrm{~Hz}, 2$ $\left.\mathrm{x}-\mathrm{CH}_{3}\right) ;{ }^{13} \mathrm{C} \mathrm{NMR}\left(\mathrm{CDCl}_{3}, 75 \mathrm{MHz}\right): \delta 158.8,138.2,130.0,129.9,129.1,128.7,115.6,80.8$, 74.2, 73.1, 71.9, 70.0, 61.2, 55.6, 34.6, 25.2, 17.9, -5.7; HRMS (ESI): $\mathrm{m} / \mathrm{z}$ calculated for $\mathrm{C}_{26} \mathrm{H}_{40} \mathrm{O}_{5} \mathrm{NaSi}[\mathrm{M}+\mathrm{Na}]^{+}$483.1603, found 483.1618 .

\section{((3R,4R)-4-(Benzyloxy)-3-methoxy-5-(4-methoxybenzyloxy)pentyloxy)(tert.-} butyl)dimethylsilane (9):

To an ice cooled suspension of $\mathrm{NaH}(0.73 \mathrm{~g}, 30.65 \mathrm{mmol})$ in THF $(50 \mathrm{~mL})$, alcohol 11 (4.7 g, $10.21 \mathrm{mmol}$ ) was added, stirred for $30 \mathrm{~min}$ and treated with a solution of $\mathrm{CH}_{3} \mathrm{I}$ $(0.82 \mathrm{~mL}, 13.28 \mathrm{mmol})$ in $\mathrm{THF}(25 \mathrm{~mL})$ and stirred at $35^{\circ} \mathrm{C}$ temperature for $6 \mathrm{~h}$. Work up as described for 14 and purification of the residue by column chromatography (60-120 mesh silica gel, 8\% EtOAc in pet. ether) to furnish 9 (4.1 g, 85\%) as a yellow liquid with 100\% diastereoselectivity. $[\alpha]_{\mathrm{D}}^{25}=+91.8\left(\mathrm{c} 0.5, \mathrm{CHCl}_{3}\right)$; IR (neat): 3451, 2929, 2857, 1612, 1513,

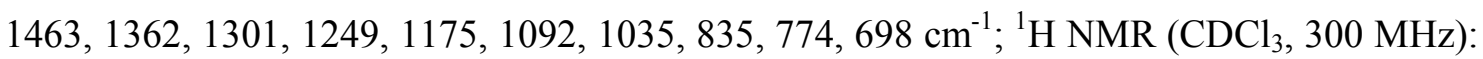
$\delta$ 7.37-7.28 (m, 5H, ArH-Bn), 7.25 (d, 2H, $J=8.30 \mathrm{~Hz}, \operatorname{ArH}-\mathrm{PMB}), 6.87$ (d, 2H, $J=8.30 \mathrm{~Hz}$, ArH-PMB), 4.75-4.60 (m, 2H, benzylic), 4.50-4.44 (m, 2H, benzylic), 3.80 (s, 3H, $\left.-\mathrm{OCH}_{3}\right)$, 3.75-3.48 (m, 6H, 2 x - $\left.-\mathrm{OCH}_{2}, 2 \mathrm{x}-\mathrm{OCH}\right), 3.38\left(\mathrm{~s}, 3 \mathrm{H},-\mathrm{OCH}_{3}\right), 1.82-1.56\left(\mathrm{~m}, 2 \mathrm{H},-\mathrm{CH}_{2}\right), 0.88$ (s, 9H, $\left.3 \mathrm{x}-\mathrm{CH}_{3}\right), 0.04\left(\mathrm{~s}, 6 \mathrm{H}, 2 \mathrm{x}-\mathrm{CH}_{3}\right) ;{ }^{13} \mathrm{C} \mathrm{NMR}\left(\mathrm{CDCl}_{3}, 75 \mathrm{MHz}\right): \delta 159.1,138.7,129.0$, $128.1,127.8,127.5,127.3,113.6,79.0,77.8,76.9,72.9,70.0,59.4,58.6,55.1,33.3,25.8$, 18.1, -5.4; HRMS (ESI): $\mathrm{m} / \mathrm{z}$ calculated for $\mathrm{C}_{27} \mathrm{H}_{42} \mathrm{O}_{5} \mathrm{NaSi}[\mathrm{M}+\mathrm{Na}]^{+}$497.1836, found 497.1842 .

(2R,3R)-2-(Benzyloxy)-5-(tert.-butyldimethylsilyloxy)-3-methoxypentan-1-ol (20): 
To a solution of $9(4 \mathrm{~g}, 8.43 \mathrm{mmol})$ in aq. $\mathrm{CH}_{2} \mathrm{Cl}_{2}(40 \mathrm{~mL}, 19: 1)$, DDQ (2.87 g, 12.65 mmol) was added and stirred at $35{ }^{\circ} \mathrm{C}$ temperature for $1 \mathrm{~h}$. The reaction mixture was quenched with sat. $\mathrm{NaHCO}_{3}$ solution $(25 \mathrm{~mL})$, filtered, washed with $\mathrm{CH}_{2} \mathrm{Cl}_{2}(40 \mathrm{~mL})$. The filtrate was washed with water $(40 \mathrm{~mL})$, brine $(40 \mathrm{~mL})$, dried $\left(\mathrm{Na}_{2} \mathrm{SO}_{4}\right)$ and evaporated under reduced pressure. The residue was purified by column chromatography (60-120 mesh silica gel, 14\% EtOAc in pet. ether) to furnish $20(2.6 \mathrm{~g}, 87 \%)$ as a yellow liquid with $100 \%$ diastereoselectivity. $[\alpha]^{25}=+100.5\left(c 0.5, \mathrm{CHCl}_{3}\right)$; IR (neat): 3418, 2926, 1713, 1607, 1454, $1375,1273,1174,1065,769,710 \mathrm{~cm}^{-1} ;{ }^{1} \mathrm{H}$ NMR $\left(\mathrm{CDCl}_{3}, 300 \mathrm{MHz}\right): \delta 7.39-7.29(\mathrm{~m}, 5 \mathrm{H}$, ArH-Bn), 4.91-4.75 (m, 2H, benzylic), 4.08-3.90 (m, 2H, $\left.-\mathrm{OCH}_{2}\right), 3.87$ (s, 3H, $\left.-\mathrm{OCH}_{3}\right), 3.83-$ $3.68\left(\mathrm{~m}, 4 \mathrm{H},-\mathrm{OCH}_{2}, 2 \mathrm{x}-\mathrm{OCH}\right), 2.22-1.95\left(\mathrm{~m}, 2 \mathrm{H},-\mathrm{CH}_{2}\right), 0.95\left(\mathrm{~s}, 9 \mathrm{H}, 3 \mathrm{x}-\mathrm{CH}_{3}\right), 0.08(\mathrm{~s}, 6 \mathrm{H}$, $\left.2 \mathrm{x}-\mathrm{CH}_{3}\right) ;{ }^{13} \mathrm{C} \mathrm{NMR}\left(\mathrm{CDCl}_{3}, 75 \mathrm{MHz}\right): \delta 137.9,128.5,128.0,127.9,127.8,127.7,80.6,79.4$, 72.9, 61.6, 60.5, 58.6, 40.3, 29.6, 14.3, -3.6; HRMS (ESI): $\mathrm{m} / \mathrm{z}$ calculated for $\mathrm{C}_{19} \mathrm{H}_{34} \mathrm{O}_{4} \mathrm{NaSi}$ $[\mathrm{M}+\mathrm{Na}]^{+}$377.1228, found 377.1236.

\section{$(5 S, 6 S, 7 R)-5$-Allyl-6-(benzyloxy)-7-methoxy-11,11,12,12-tetramethyl-2,4,10-trioxa-11-}

\section{silatridecane (22):}

To a cooled $\left(0{ }^{\circ} \mathrm{C}\right)$ solution of $21(2.1 \mathrm{~g}, 5.32 \mathrm{mmol})$ in $\mathrm{CH}_{2} \mathrm{Cl}_{2}(20 \mathrm{~mL})$, DIPEA (4.6 $\mathrm{mL}, 26.64 \mathrm{mmol})$ and $\mathrm{MOM}-\mathrm{Cl}(0.5 \mathrm{~mL}, 6.39 \mathrm{mmol})$ were added sequentially and stirred at $35{ }^{\circ} \mathrm{C}$ temperature for $6 \mathrm{~h}$. The reaction mixture was evaporated and purified the residue by column chromatography (60-120 mesh silica gel, 6\% EtOAc in pet. ether) to afford 22 (2 g, $86 \%$ ) as a yellow liquid with $100 \%$ diastereoselectivity. $[\alpha]^{25}=+75.6\left(c\right.$ o.5, $\left.\mathrm{CHCl}_{3}\right)$; IR (neat): $3447,3070,2929,2855,1723,1604,1451,1375,1314,1272,1152,1102,1033,918$, 837, $775 \mathrm{~cm}^{-1}$; ${ }^{1} \mathrm{H}$ NMR (CDCl 3 , $\left.300 \mathrm{MHz}\right): \delta$ 7.39-7.27 (m, 5H, ArH-Bn), 5.94-5.85 (m, 1H, olefinic), $5.14-5.07$ (m, 2H, olefinic), 4.75-4.63 (m, 4H, benzylic, $\left.-\mathrm{OCH}_{2}\right), 3.77-3.69(\mathrm{~m}, 3 \mathrm{H}$, $\left.-\mathrm{OCH}_{2},-\mathrm{OCH}\right), 3.66-3.54(\mathrm{~m}, 2 \mathrm{H}, 2 \mathrm{x}-\mathrm{OCH}), 3.39\left(\mathrm{~s}, 3 \mathrm{H},-\mathrm{OCH}_{3}\right), 3.20\left(\mathrm{~s}, 3 \mathrm{H},-\mathrm{OCH}_{3}\right)$, 2.52-2.45 (m, 2H, $\left.-\mathrm{CH}_{2}\right), 1.87-1.73\left(\mathrm{~m}, 2 \mathrm{H},-\mathrm{CH}_{2}\right), 0.91\left(\mathrm{~s}, 9 \mathrm{H}, 3 \mathrm{x}-\mathrm{CH}_{3}\right), 0.06(\mathrm{~s}, 6 \mathrm{H}, 2 \mathrm{x}-$ 
$\left.\mathrm{CH}_{3}\right) ;{ }^{13} \mathrm{C} \mathrm{NMR}\left(\mathrm{CDCl}_{3}, 75 \mathrm{MHz}\right): \delta 138.7,135.1,128.2,127.6,127.4,126.6,117.1,96.3$, 80.4, 78.3, 73.4, 59.6, 59.4, 57.7, 56.6, 55.8, 41.3, 35.4, 33.4, 25.9, 18.3, -5.3; HRMS (ESI): $\mathrm{m} / \mathrm{z}$ calculated for $\mathrm{C}_{24} \mathrm{H}_{42} \mathrm{O}_{5} \mathrm{NaSi}[\mathrm{M}+\mathrm{Na}]^{+} 461.1827$, found 461.1832 .

\section{(3R,4S,5S)-4-(Benzyloxy)-3-methoxy-5-(methoxymethoxy)oct-7-en-1-ol (23):}

To a solution of $22(1.9 \mathrm{~g}, 4.33 \mathrm{mmol})$ in THF $(20 \mathrm{~mL})$ at $0{ }^{\circ} \mathrm{C}, 1 \mathrm{M}$ solution of TBAF in THF (5.2 mL, $5.2 \mathrm{mmol}$ ) was added and stirred for $1 \mathrm{~h}$. Work up as described for $\mathbf{1 5}$ and purification of the residue by column chromatography (60-120 mesh silica gel, 14\% EtOAc in pet. ether) to give $\mathbf{2 3}(1.25 \mathrm{~g}, 89 \%)$ as a yellow liquid with $100 \%$ diastereoselectivity. $[\alpha]_{\mathrm{D}}^{25}=+116.2\left(c\right.$ 0.5, $\left.\mathrm{CHCl}_{3}\right)$; IR (neat): 3448, 3070, 2929, 1723, 1640, 1452, 1271, 1211, 1102, 1034, 916, $739 \mathrm{~cm}^{-1} ;{ }^{1} \mathrm{H}$ NMR $\left(\mathrm{CDCl}_{3}, 300 \mathrm{MHz}\right): \delta$ 7.36-7.27 (m, 5H, ArH-Bn), 5.95$5.81(\mathrm{~m}, 1 \mathrm{H}$, olefinic), $5.16-5.07$ (m, 2H, olefinic), 4.80-4.69 (m, 2H, benzylic), $4.67(\mathrm{~s}, 2 \mathrm{H}$, $\left.-\mathrm{OCH}_{2}\right), 3.82-3.65\left(\mathrm{~m}, 5 \mathrm{H},-\mathrm{OCH}_{2}, 3 \times-\mathrm{OCH}\right), 3.41\left(\mathrm{~s}, 3 \mathrm{H},-\mathrm{OCH}_{3}\right), 3.38\left(\mathrm{~s}, 3 \mathrm{H},-\mathrm{OCH}_{3}\right)$, 2.70-2.38 (m, 2H, $\left.-\mathrm{CH}_{2}\right), 1.98-1.75\left(\mathrm{~m}, 2 \mathrm{H},-\mathrm{CH}_{2}\right) ;{ }^{13} \mathrm{C} \mathrm{NMR}\left(\mathrm{CDCl}_{3}, 75 \mathrm{MHz}\right): \delta 138.3$, $134.2,128.4,128.3,127.9,127.8,127.6,117.7,96.9,81.0,79.7,74.4,60.4,57.1,55.8,36.2$, 32.0, 29.7; HRMS (ESI): $\mathrm{m} / \mathrm{z}$ calculated for $\mathrm{C}_{18} \mathrm{H}_{28} \mathrm{O}_{5} \mathrm{Na}[\mathrm{M}+\mathrm{Na}]^{+} 347.0812$, found 347.0820.

\section{(3R,4S,5S)-4-(Benzyloxy)-3-methoxy-5-(methoxymethoxy)oct-7-enoic acid (5):}

To a stirred solution of $23(1.0 \mathrm{~g}, 3.08 \mathrm{mmol})$ in $\mathrm{CH}_{2} \mathrm{Cl}_{2}: \mathrm{H}_{2} \mathrm{O}(1: 1,10 \mathrm{~mL})$, TEMPO (0.14 g, $0.92 \mathrm{mmol})$ and BAIB $(2.98 \mathrm{~g}, 9.25 \mathrm{mmol})$ were added at $0{ }^{\circ} \mathrm{C}$ and stirred for $2 \mathrm{~h}$. The reaction mixture was diluted with water $(10 \mathrm{~mL})$ and extracted with $\mathrm{CH}_{2} \mathrm{Cl}_{2}(2 \times 20 \mathrm{~mL})$. Organic layers were washed with sat. aq. hypo $(15 \mathrm{~mL})$, brine $(20 \mathrm{~mL})$ and dried $\left(\mathrm{Na}_{2} \mathrm{SO}_{4}\right)$. Solvent was evaporated and purified the residue by column chromatography (60-120 mesh silica gel, 26\% EtOAc in pet. ether) to give acid $5(0.84 \mathrm{~g}, 81 \%)$ as a colorless gummy oil with $100 \%$ diastereoselectivity. $[\alpha]^{25}=+126.9\left(c 0.5, \mathrm{CHCl}_{3}\right)$; IR (neat): $3451,2927,2854$, $1726,1638,1457,1380,1270,1211,1153,1101,1034,917,768,701 \mathrm{~cm}^{-1} ;{ }^{1} \mathrm{H}$ NMR 
$\left(\mathrm{CDCl}_{3}, 300 \mathrm{MHz}\right): \delta$ 7.34-7.27 (m, 5H, ArH-Bn), 5.89-5.81 (m, 1H, olefinic), 5.14 -5.08 (m, $2 \mathrm{H}$, olefinic), 4.74-4.68 (m, 2H, benzylic), $4.67\left(\mathrm{~s}, 2 \mathrm{H},-\mathrm{OCH}_{2}\right), 3.96-3.91(\mathrm{~m}, 1 \mathrm{H},-\mathrm{OCH})$, 3.80-3.77 (m, 1H, -OCH), $3.40\left(\mathrm{~s}, 3 \mathrm{H},-\mathrm{OCH}_{3}\right), 3.38\left(\mathrm{~s}, 3 \mathrm{H},-\mathrm{OCH}_{3}\right), 2.75-2.66(\mathrm{~m}, 2 \mathrm{H}$, $\left.\mathrm{CH}_{2}\right), 2.46-2.43\left(\mathrm{~m}, 2 \mathrm{H},-\mathrm{CH}_{2}\right) ;{ }^{13} \mathrm{C} \mathrm{NMR}\left(\mathrm{CDCl}_{3}, 75 \mathrm{MHz}\right): \delta 177.1,138.2,134.6,128.2$, $127.8,127.5,117.4,96.0,79.6,78.2,73.4,57.7,55.9,35.8,35.4 ;$ HRMS (ESI): $\mathrm{m} / \mathrm{z}$ calculated for $\mathrm{C}_{18} \mathrm{H}_{26} \mathrm{O}_{6} \mathrm{Na}[\mathrm{M}+\mathrm{Na}]^{+}$361.0948, found 361.0941 .

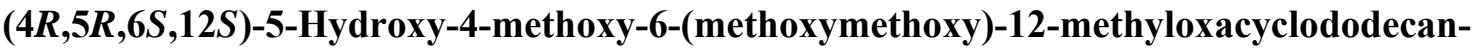
2-one (25):

To a stirred solution of $24(0.2 \mathrm{~g}, 0.51 \mathrm{mmol})$ in $\mathrm{MeOH}(10 \mathrm{~mL}), 10 \% \mathrm{Pd}-\mathrm{C}$ was added and stirred under $\mathrm{H}_{2}$ atmosphere for $12 \mathrm{~h}$. The reaction mixture was filtered through celite, concentrated the filtrate and purified the residue by column chromatography (60-120 mesh silica gel, 18\% EtOAc in pet. ether) to afford $25(0.12 \mathrm{~g}, 77 \%)$ as colorless syrup with $100 \%$ diastereoselectivity. $[\alpha]_{\mathrm{D}}^{25}=+41.6\left(\right.$ c $\left.0.5, \mathrm{CHCl}_{3}\right)$; IR (neat): 3453, 2928, 2856, 1786, $1717,1633,1461,1370,1275,1168,1104,1034,920,836,757,713 \mathrm{~cm}^{-1} ;{ }^{1} \mathrm{H}$ NMR (300 $\left.\mathrm{MHz}, \mathrm{CDCl}_{3}\right): \delta$ 4.71-4.60 (m, 3H, $\left.-\mathrm{OCH}_{2},-\mathrm{OCH}\right), 4.50-4.39(\mathrm{~m}, 1 \mathrm{H},-\mathrm{OCH}), 4.21-4.07$ (m, $1 \mathrm{H},-\mathrm{OCH}), 3.90-3.71(\mathrm{~m}, 1 \mathrm{H},-\mathrm{OCH}), 3.35\left(\mathrm{~s}, 6 \mathrm{H}, 2 \mathrm{x}-\mathrm{OCH}_{3}\right), 2.89-2.70(\mathrm{~m}, 1 \mathrm{H},-\mathrm{CH})$, 2.51-2.48 (m, 1H, -CH), 1.79-1.30 (m, 7H, 7 x -CH), $1.20\left(\mathrm{~d}, 3 \mathrm{H}, J=6.3 \mathrm{~Hz},-\mathrm{CH}_{3}\right), 0.99-$

0.77 (m, 3H, 3 x -CH); ${ }^{13} \mathrm{C}$ NMR (75 $\left.\mathrm{MHz}, \mathrm{CHCl}_{3}\right): \delta 175.5,96.5,86.4,76.2,67.9,56.4$, 55.9, 39.0, 35.3, 30.6, 29.6, 29.5, 25.5, 25.3, 23.5; HRMS (ESI): $\mathrm{m} / \mathrm{z}$ calculated for $\mathrm{C}_{15} \mathrm{H}_{28} \mathrm{O}_{6} \mathrm{Na}[\mathrm{M}+\mathrm{Na}]^{+}$327.0649, found 327.0655.

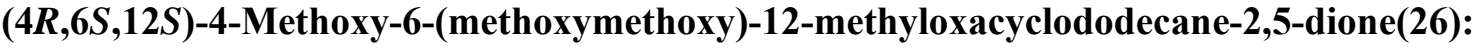

To a stirred solution of alcohol $25(0.07 \mathrm{~g}, 0.23 \mathrm{mmol})$ in dry $\mathrm{CH}_{2} \mathrm{Cl}_{2}(10 \mathrm{~mL})$, DessMartin periodinane $(0.11 \mathrm{~g}, 0.27 \mathrm{mmol})$ was added at $0{ }^{\circ} \mathrm{C}$, and stirred at $35^{\circ} \mathrm{C}$ temperature for $4 \mathrm{~h}$. The reaction mixture was quenched with a 1:1 mixture of saturated solutions of 
$\mathrm{Na}_{2} \mathrm{~S}_{2} \mathrm{O}_{3}$ and $\mathrm{NaHCO}_{3}(20 \mathrm{~mL})$, and extracted with $\mathrm{CH}_{2} \mathrm{Cl}_{2}(2 \times 20 \mathrm{~mL})$. The combined organic layers were washed with water $(20 \mathrm{~mL})$, brine $(20 \mathrm{~mL})$ and dried $\left(\mathrm{Na}_{2} \mathrm{SO}_{4}\right)$, solvent was evaporated and purified the residue by column chromatography (60-120 mesh silica gel, $15 \%$ EtOAc in pet. ether) to afford $26(0.055 \mathrm{~g}, 80 \%)$ as a yellow liquid with $100 \%$ diastereoselectivity. $[\alpha]^{25}{ }_{\mathrm{D}}=-5.8\left(c\right.$ 0.5, $\left.\mathrm{CHCl}_{3}\right)$; IR (neat): 3451, 2923, 2853, 1781, 1710, 1636, 1459, 1364, 1169, 1096, 1037, 918, $768 \mathrm{~cm}^{-1} ;{ }^{1} \mathrm{H}$ NMR (300 MHz, $\left.\mathrm{CDCl}_{3}\right): \delta$ 4.79$4.71\left(\mathrm{~m}, 3 \mathrm{H},-\mathrm{OCH}_{2},-\mathrm{OCH}\right), 4.53(\mathrm{~d}, 1 \mathrm{H}, J=8.9 \mathrm{~Hz},-\mathrm{OCH}), 4.27(\mathrm{~d}, 1 \mathrm{H}, J=5.9 \mathrm{~Hz},-$ $\mathrm{OCH}), 3.49\left(\mathrm{~s}, 3 \mathrm{H},-\mathrm{OCH}_{3}\right), 3.47\left(\mathrm{~s}, 3 \mathrm{H},-\mathrm{OCH}_{3}\right), 2.97-2.90(\mathrm{~m}, 1 \mathrm{H},-\mathrm{CH}), 2.63(\mathrm{dd}, 1 \mathrm{H}, J=$ 8.5 Hz, 13.6 Hz, -CH), 2.10-2.01 (m, 1H, -CH), 1.69-1.58 (m, 2H, 2 x -CH), 1.57-1.48 (m, $2 \mathrm{H}, 2 \mathrm{x}-\mathrm{CH}), 1.47-1.38(\mathrm{~m}, 2 \mathrm{H}, 2 \mathrm{x}-\mathrm{CH}), 1.36-1.25(\mathrm{~m}, 2 \mathrm{H}, 2 \mathrm{x}-\mathrm{CH}), 1.23(\mathrm{~d}, 3 \mathrm{H}, J=6.4$ $\left.\mathrm{Hz},-\mathrm{CH}_{3}\right), 1.09-1.07(\mathrm{~m}, 1 \mathrm{H},-\mathrm{CH}) ;{ }^{13} \mathrm{C} \mathrm{NMR}\left(75 \mathrm{MHz}, \mathrm{CHCl}_{3}\right): \delta 208.8,170.6,76.1,74.4$, 73.3, 58.4, 41.4, 33.4, 30.0, 26.5, 23.8, 22.2, 20.8; HRMS (ESI): $\mathrm{m} / \mathrm{z}$ calculated for $\mathrm{C}_{15} \mathrm{H}_{26} \mathrm{O}_{6} \mathrm{Na}[\mathrm{M}+\mathrm{Na}]^{+} 325.1319$, found 3251327 .

\section{(2R,3R)-2-(Benzyloxy)-5-(tert.-butyldimethylsilyloxy)pentane-1,3-diol(11a):}

To a solution of $\mathbf{1 1}(0.6 \mathrm{~g}, 1.30 \mathrm{mmol})$ in aq. $\mathrm{CH}_{2} \mathrm{Cl}_{2}(6 \mathrm{~mL}, 19: 1)$, DDQ (0.44 g, 1.95 mmol) was added and stirred at $35{ }^{\circ} \mathrm{C}$ temperature for $1 \mathrm{~h}$. Work up as described for $\mathbf{2 0}$ and purification of the residue by column chromatography (60-120 mesh silica gel, 24\% EtOAc in pet. ether) furnished $11 \mathrm{a}(0.38 \mathrm{~g}, 85 \%)$ as a colorless liquid with $100 \%$ diastereoselectivity. $[\alpha]^{25}=+80.1\left(c\right.$ 0.5, $\mathrm{CHCl}_{3}$ ); IR (neat): 3460, 3420, 3065, 2938, 1715, 1640, 1470, 1375, 1310, 1173, 1095, 1020, 919, $757 \mathrm{~cm}^{-1} ;{ }^{1} \mathrm{H}$ NMR $\left(\mathrm{CDCl}_{3}, 300 \mathrm{MHz}\right): \delta$ 7.37-7.27 (m, 5H, ArH-Bn), 4.67 (s, 2H, benzylic), 4.24-4.16 (m, 1H, -OCH), 3.87-3.65 (m, 4H, 2 x -OCH, $\left.\mathrm{OCH}_{2}\right), 3.62-3.56(\mathrm{~m}, 1 \mathrm{H},-\mathrm{OCH}), 2.03-1.77\left(\mathrm{~m}, 2 \mathrm{H},-\mathrm{CH}_{2}\right), 0.93\left(\mathrm{~s}, 9 \mathrm{H}, 3 \mathrm{x}-\mathrm{CH}_{3}\right), 0.12(\mathrm{~s}$, $\left.6 \mathrm{H}, 2 \mathrm{x}-\mathrm{CH}_{3}\right) ;{ }^{13} \mathrm{C} \mathrm{NMR}\left(\mathrm{CDCl}_{3}, 75 \mathrm{MHz}\right): \delta 134.7,128.4,127.8,127.6,83.2,74.3,71.1$, 59.0, 55.9, 35.2, 25.8, 17.9, -4.3, -4.7; HRMS (ESI): $\mathrm{m} / \mathrm{z}$ calculated for $\mathrm{C}_{18} \mathrm{H}_{32} \mathrm{O}_{4} \mathrm{NaSi}$ $[\mathrm{M}+\mathrm{Na}]^{+}$363.2071, found 363.2079. 
(3R,4R,5S)-4-(Benzyloxy)-1-(tert.-butyldimethylsilyloxy)oct-7-ene-3,5-diol (28):

To a solution of alcohol 11a $(0.3 \mathrm{~g}, 0.88 \mathrm{mmol})$ in $\mathrm{CH}_{2} \mathrm{Cl}_{2}(10 \mathrm{~mL}), \mathrm{P}^{\mathrm{H}} 8.6$ buffer $(2$ $\mathrm{mL}$ ) (aq. solutions of $0.5 \mathrm{M} \mathrm{NaHCO}_{3}, 0.05 \mathrm{M} \mathrm{K}_{2} \mathrm{CO}_{3}$ ), TEMPO (13 mg, $0.1 \mathrm{mmol}$ ), TBACl (24 mg, $0.08 \mathrm{mmol}), N$-Chlorosuccinimide $(0.11 \mathrm{~g}, 0.879 \mathrm{mmol})$ were added, stirred at $35^{\circ} \mathrm{C}$ temperature for $1 \mathrm{~h}$. After completion of the reaction, it was diluted with water $(3 \mathrm{~mL})$ and extracted with $\mathrm{CH}_{2} \mathrm{Cl}_{2}(2 \times 5 \mathrm{~mL})$. Organic layers were washed with brine $(5 \mathrm{~mL})$, dried $\left(\mathrm{Na}_{2} \mathrm{SO}_{4}\right)$, evaporated to furnish the corresponding aldehyde 27.

To a solution of the aldehyde $27(0.25 \mathrm{~g}, 0.74 \mathrm{mmol})$ in $\mathrm{CH}_{2} \mathrm{Cl}_{2}(3 \mathrm{~mL}), \mathrm{MgBr}_{2} \cdot \mathrm{OEt}_{2}$ $(0.38 \mathrm{~g}, 1.47 \mathrm{mmol})$ was added at $-78^{\circ} \mathrm{C}$ under nitrogen atmosphere. The reaction mixture was stirred at $-78{ }^{\circ} \mathrm{C}$ for $45 \mathrm{~min}$. Allyltributyltin $(0.3 \mathrm{~mL}, 1.11 \mathrm{mmol})$ was added to the reaction mixture dropwise, and stirred at $-78{ }^{\circ} \mathrm{C}$ for $4 \mathrm{~h}$. Work up as described for 21 and purification of the residue by column chromatography (60-120 mesh silica gel, 16\% EtOAc in pet. ether) to furnish $\mathbf{2 8}(0.22 \mathrm{~g}, 79 \%)$ as a colorless syrup. The diasterioselectivity of syn and anti alcohols are 96:4. $[\alpha]^{25}{ }_{\mathrm{D}}=+81.6\left(c\right.$ 0.5, $\left.\mathrm{CHCl}_{3}\right)$; IR (neat): 3420, 3060, 2935, 1710, $1642,1450,1376,1316,1250,1176,1099,1026,919,758 \mathrm{~cm}^{-1} ;{ }^{1} \mathrm{H}$ NMR $\left(\mathrm{CDCl}_{3}, 300\right.$ MHz): $\delta$ 7.48-7.28 (m, 5H, ArH-Bn), 6.06-5.90 (m, 1H, olefinic), $5.26-5.17$ (m, 2H, olefinic), $4.74\left(\mathrm{~s}, 2 \mathrm{H}\right.$, benzylic), 4.33-4.25 (m, 1H, -OCH), 3.92-3.76 (m, 3H, -OCH, $\left.-\mathrm{OCH}_{2}\right)$, 3.71-3.68 (m, 1H, -OCH), 2.72-2.47 (m, 2H, $\left.-\mathrm{CH}_{2}\right), 2.13-1.85\left(\mathrm{~m}, 2 \mathrm{H},-\mathrm{CH}_{2}\right), 1.10(\mathrm{~s}, 9 \mathrm{H}, 3 \mathrm{x}$ $\left.-\mathrm{CH}_{3}\right), 0.21\left(\mathrm{~s}, 6 \mathrm{H}, 2 \mathrm{x}-\mathrm{CH}_{3}\right) ;{ }^{13} \mathrm{C} \mathrm{NMR}\left(\mathrm{CDCl}_{3}, 75 \mathrm{MHz}\right): \delta 138.0,134.4,128.1,127.6$, $127.5,117.2,83.4,74.6,70.1,60.0,59.7,36.3,35.4,26.4,-5.7,-5.4 ;$ HRMS (ESI): $m / z$ calculated for $\mathrm{C}_{21} \mathrm{H}_{36} \mathrm{O}_{4} \mathrm{NaSi}[\mathrm{M}+\mathrm{Na}]^{+}$403.2476, found 403.2482.

(2-((4R,5R,6S)-6-Allyl-5-(benzyloxy)-2,2-dimethyl-1,3-dioxan-4-yl)ethoxy)(tert.butyl)dimethylsilane (29): 
To a cooled $\left(0{ }^{\circ} \mathrm{C}\right)$ solution of $28(0.15 \mathrm{~g}, 0.39 \mathrm{mmol})$ in dry $\mathrm{CH}_{2} \mathrm{Cl}_{2}(3 \mathrm{~mL}), 2$, 2 dimethoxy propane $(0.1 \mathrm{~mL}, 0.78 \mathrm{mmol})$, PTSA (cat.) were added and stirred at $35{ }^{\circ} \mathrm{C}$ temperature for $1 \mathrm{~h}$. The reaction mixture was neutralised with $\mathrm{Et}_{3} \mathrm{~N}(1 \mathrm{~mL})$ and extracted with $\mathrm{CH}_{2} \mathrm{Cl}_{2}(2 \times 3 \mathrm{~mL})$. The organic layers were washed with water $(3 \mathrm{~mL})$, brine $(3 \mathrm{~mL})$ and dried $\left(\mathrm{Na}_{2} \mathrm{SO}_{4}\right)$. Solvent was evaporated and purified the residue by column chromatography (60-120 mesh silica gel, 11\% EtOAc in pet. ether) to furnish 29 (0.12 g, $73 \%)$ as a yellow liquid with $100 \%$ diastereoselectivity. $[\alpha]^{25}=+86.9\left(c\right.$ o.5, $\left.\mathrm{CHCl}_{3}\right)$; IR (neat): $3340,2943,2861,1682,1520,1374,1250,1105,1020,825,775 \mathrm{~cm}^{-1} ;{ }^{1} \mathrm{H}$ NMR (300 $\left.\mathrm{MHz}, \mathrm{CDCl}_{3}\right): \delta$ 7.39-7.28 (m, 5H, ArH-Bn), 6.08-5.98 (m, 1H, Olefinic), 5.30-5.22 (m, 2H, Olefinic), 4.80-4.74 (m, 2H, benzylic), 4.30-4.24 (m, 1H, -OCH), 3.97-3.80 (m, 3H, -OCH, $\left.\mathrm{OCH}_{2}\right), 3.71-3.68(\mathrm{~m}, 1 \mathrm{H},-\mathrm{OCH}), 2.69-2.41\left(\mathrm{~m}, 2 \mathrm{H},-\mathrm{CH}_{2}\right), 2.10-1.93\left(\mathrm{~m}, 2 \mathrm{H},-\mathrm{CH}_{2}\right), 1.54(\mathrm{~s}$, $\left.3 \mathrm{H},-\mathrm{CH}_{3}\right), 1.28\left(\mathrm{~s}, 3 \mathrm{H},-\mathrm{CH}_{3}\right), 1.07\left(\mathrm{~s}, 9 \mathrm{H}, 3 \mathrm{x}-\mathrm{CH}_{3}\right), 0.20\left(\mathrm{~s}, 6 \mathrm{H}, 2 \mathrm{x}-\mathrm{CH}_{3}\right) ;{ }^{13} \mathrm{C} \mathrm{NMR}(75$ $\left.\mathrm{MHz}, \mathrm{CDCl}_{3}\right): \delta 139.1,135.4,128.6,127.2,127.0,117.1,96.4,80.7,78.1,73.5,59.7,41.6$, 35.3, 33.4, 30.2, 26.5, 19.7, 18.2, -5.4; HRMS (ESI): $\mathrm{m} / \mathrm{z}$ calculated for $\mathrm{C}_{24} \mathrm{H}_{40} \mathrm{O}_{4} \mathrm{NaSi}$ $[\mathrm{M}+\mathrm{Na}]^{+}$443.1685, found 443.1690.

\section{(R)-5-((R)-1-(Benzyloxy)-2-(4-methoxybenzyloxy)ethyl)-2,2,3,3,9,9,10,10-octamethyl-4,8-} dioxa-3,9-disilaundecane (10):

To a cooled $\left(0{ }^{\circ} \mathrm{C}\right)$ solution of $11(4 \mathrm{~g}, 8.69 \mathrm{mmol})$ in $\mathrm{CH}_{2} \mathrm{Cl}_{2}(40 \mathrm{~mL})$, imidazole (1.77 g, $26.02 \mathrm{mmol})$ was added. After $30 \mathrm{~min}$ TBDMS-Cl (1.3 g, $8.66 \mathrm{mmol})$ was added and stirred at $35^{\circ} \mathrm{C}$ temperature for $2 \mathrm{~h}$. The reaction mixture was evaporated and purified the residue by column chromatography (60-120 mesh silica gel, $8 \%$ EtOAc in pet. ether) to afford $10(4.2 \mathrm{~g}, 84 \%)$ as a yellow liquid with $100 \%$ diastereoselectivity. $[\alpha]^{25}=+113.6(\mathrm{c}$ 0.5, $\mathrm{CHCl}_{3}$ ); IR (neat): 2952, 2925, 2857, 1716, 1610, 1512, 1470, 1390, 1261, 1097, 1034, $771 \mathrm{~cm}^{-1} ;{ }^{1} \mathrm{H}$ NMR $\left(\mathrm{CDCl}_{3}, 300 \mathrm{MHz}\right): \delta$ 7.40-7.33 (m, 5H, ArH-Bn), $7.28(\mathrm{~d}, 2 \mathrm{H}, J=8.8$ Hz, ArH-PMB), 6.90 (d, 2H, $J=8.5 \mathrm{~Hz}$, ArH-PMB), 4.78-4.67 (m, 2H, benzylic), 4.55-4.45 
(m, 2H, benzylic), 4.10-4.01 (m, 1H, -OCH), $3.85\left(\mathrm{~s}, 3 \mathrm{H},-\mathrm{OCH}_{3}\right), 3.79-3.57(\mathrm{~m}, 5 \mathrm{H}, 2 \mathrm{x}-$ $\left.\mathrm{OCH}_{2},-\mathrm{OCH}\right), 1.96-1.67\left(\mathrm{~m}, 2 \mathrm{H},-\mathrm{CH}_{2}\right), 0.93-0.91\left(\mathrm{~m}, 18 \mathrm{H}, 6 \mathrm{x}-\mathrm{CH}_{3}\right), 0.09(\mathrm{~d}, 6 \mathrm{H}, J=3.3$ $\left.\mathrm{Hz}, 2 \mathrm{x}-\mathrm{CH}_{3}\right), 0.07\left(\mathrm{~s}, 6 \mathrm{H}, 2 \mathrm{x}-\mathrm{CH}_{3}\right) ;{ }^{13} \mathrm{C} \mathrm{NMR}\left(\mathrm{CDCl}_{3}, 75 \mathrm{MHz}\right): \delta 159.0,138.9,130.6$, $129.1,128.1,127.7,127.6,127.3,113.6,82.1,72.9,72.3,70.3,68.1,59.6,55.2,34.7,25.9$, 18.2, 17.9, -4.6, -5.3; HRMS (ESI): $\mathrm{m} / \mathrm{z}$ calculated for $\mathrm{C}_{32} \mathrm{H}_{54} \mathrm{O}_{5} \mathrm{NaSi}_{2}[\mathrm{M}+\mathrm{Na}]^{+}$597.2429, found 597.2436.

\section{(2R,3R)-2-(Benzyloxy)-3,5-bis(tert.-butyldimethylsilyloxy)pentan-1-ol (30):}

To a solution of $\mathbf{1 0}(4 \mathrm{~g}, 6.96 \mathrm{mmol})$ in aq. $\mathrm{CH}_{2} \mathrm{Cl}_{2}(40 \mathrm{~mL}, 19: 1)$, DDQ (2.37 g, $10.45 \mathrm{mmol}$ ) was added and stirred at $35^{\circ} \mathrm{C}$ temperature for $1 \mathrm{~h}$. Work up as described for 20 and purification of the residue by column chromatography (60-120 mesh silica gel, 13\% EtOAc in pet. ether) to furnish $30(2.5 \mathrm{~g}, 79 \%)$ as a yellow liquid with $100 \%$ diastereoselectivity. $[\alpha]_{\mathrm{D}}^{25}=+101.4\left(\right.$ c 0.5, $\left.\mathrm{CHCl}_{3}\right)$; IR (neat): 3449, 3065, 3031, 2954, 2930, $2887,2857,1725,1609,1467,1388,1361,1254,1095,936,776,698 \mathrm{~cm}^{-1} ;{ }^{1} \mathrm{H}$ NMR $\left(\mathrm{CDCl}_{3}, 300 \mathrm{MHz}\right): \delta$ 7.37-7.29 (m, 5H, ArH-Bn), 4.76-4.53 (m, 2H, benzylic), 4.16-4.05 (m, $1 \mathrm{H},-\mathrm{OCH}), 3.84-3.47\left(\mathrm{~m}, 5 \mathrm{H}, 2 \mathrm{x}-\mathrm{OCH}_{2},-\mathrm{OCH}\right), 2.37-2.24(\mathrm{~m}, 1 \mathrm{H},-\mathrm{CH}), 1.82-1.67(\mathrm{~m}, 1 \mathrm{H}$, -CH), 0.94-0.85(m, 18H, 6 x - $\left.\mathrm{CH}_{3}\right), 0.11-0.02\left(\mathrm{~m}, 12 \mathrm{H}, 4 \mathrm{x}-\mathrm{CH}_{3}\right) ;{ }^{13} \mathrm{C} \mathrm{NMR}\left(\mathrm{CDCl}_{3}, 75\right.$ MHz): $\delta 138.3,132.2,128.4,127.8,127.6,114.3,80.7,72.2,68.3,59.4,59.2,37.0,25.9$, 18.2, 17.9, -4.5, -4.9, -5.3; HRMS (ESI): $\mathrm{m} / \mathrm{z}$ calculated for $\mathrm{C}_{24} \mathrm{H}_{46} \mathrm{O}_{4} \mathrm{NaSi}_{2}[\mathrm{M}+\mathrm{Na}]^{+}$ 477.2129, found 477.2135.

\section{(4S,5R,6R)-5-(Benzyloxy)-6,8-bis(tert.-butyldimethylsilyloxy)oct-1-en-4-ol (31):}

To a solution of oxalyl chloride $(0.7 \mathrm{~mL}, 7.59 \mathrm{mmol})$ in dry $\mathrm{CH}_{2} \mathrm{Cl}_{2}(10 \mathrm{~mL})$ at -78 ${ }^{\circ} \mathrm{C}$, dry DMSO (2.1 mL, $\left.15.13 \mathrm{mmol}\right)$ was added dropwise and stirred for $20 \mathrm{~min}$. A solution of $30(2.3 \mathrm{~g}, 5.06 \mathrm{mmol})$ in dry $\mathrm{CH}_{2} \mathrm{Cl}_{2}(20 \mathrm{~mL})$ was added and stirred for $2 \mathrm{~h}$ at $-78{ }^{\circ} \mathrm{C}$. Work up as described for 15a furnish the corresponding aldehyde 30a. 
To a solution of the aldehyde $\mathbf{3 0 a}(2.3 \mathrm{~g}, 5.08 \mathrm{mmol})$ in dry $\mathrm{CH}_{2} \mathrm{Cl}_{2}(25 \mathrm{~mL})$, $\mathrm{MgBr}_{2} \cdot \mathrm{OEt}_{2}(2.62 \mathrm{~g}, 10.17 \mathrm{mmol})$ was added at $-78^{\circ} \mathrm{C}$. The reaction mixture was stirred at $78^{\circ} \mathrm{C}$ for $45 \mathrm{~min}$, then allyltributyltin $(2.3 \mathrm{~mL}, 7.63 \mathrm{mmol})$ was added to the reaction mixture dropwise, and the whole was stirred for $4 \mathrm{~h}$ at $-78{ }^{\circ} \mathrm{C}$. Work up as described for 21 and purification of the residue by column chromatography (60-120 mesh silica gel, 8\% EtOAc in pet. ether) to furnish $\mathbf{3 1}(1.9 \mathrm{~g}, 76 \%)$ as a yellow syrup. The diastereo selectivity of syn and anti alcohols are 96:4. $[\alpha]^{25}{ }_{\mathrm{D}}=+84.2\left(\right.$ c 0.5, $\left.\mathrm{CHCl}_{3}\right)$; IR (neat): 3448, 3070, 2955, 2929, 2856, 1725, 1640, 1463, 1383, 1254, 1091, 914, 775, $698 \mathrm{~cm}^{-1} ;{ }^{1} \mathrm{H} \mathrm{NMR}\left(\mathrm{CDCl}_{3}, 300 \mathrm{MHz}\right)$ : א 7.40-7.29 (m, 5H, ArH-Bn), 5.90-5.71 (m, 1H, olefinic), 5.09-4.97 (m, 2H, olefinic), 4.90$4.52\left(\mathrm{~m}, 2 \mathrm{H}\right.$, benzylic), 4.33-4.09 (m,1H, -OCH), 3.93-3.62 (m, 3H, - $\left.\mathrm{OCH}_{2},-\mathrm{OCH}\right), 3.39-$ $3.27(\mathrm{~m}, 1 \mathrm{H},-\mathrm{OCH}), 2.46-2.18\left(\mathrm{~m}, 2 \mathrm{H},-\mathrm{CH}_{2}\right), 1.72-1.58\left(\mathrm{~m}, 2 \mathrm{H},-\mathrm{CH}_{2}\right), 0.97-0.85(\mathrm{~m}, 18 \mathrm{H}$, $\left.6 \mathrm{x}-\mathrm{CH}_{3}\right), 0.13-0.03\left(\mathrm{~m}, 12 \mathrm{H}, 4 \mathrm{x}-\mathrm{CH}_{3}\right) ;{ }^{13} \mathrm{C} \mathrm{NMR}\left(\mathrm{CDCl}_{3}, 75 \mathrm{MHz}\right): \delta 138.0,135.3,128.3$, $127.9,127.6,116.9,81.4,72.9,70.3,69.0,67.7,59.3,39.9,35.2,27.8,25.8,17.5,13.5,-4.3$, $-4.8,-5.3$; HRMS (ESI): $\mathrm{m} / \mathrm{z}$ calculated for $\mathrm{C}_{27} \mathrm{H}_{50} \mathrm{O}_{4} \mathrm{NaSi}_{2}[\mathrm{M}+\mathrm{Na}]^{+}$517.2048, found 517.2055 .

(5S,6R,7R)-5-Allyl-6-(benzyloxy)-7-(tert.-butyldimethylsilyloxy)-11,11,12,12tetramethyl-2,4,10-trioxa-11-silatridecane (32):

To a cooled $\left(0{ }^{\circ} \mathrm{C}\right)$ solution of $\mathbf{3 1}(1.8 \mathrm{~g}, 3.64 \mathrm{mmol})$ in $\mathrm{CH}_{2} \mathrm{Cl}_{2}(20 \mathrm{~mL})$, DIPEA (3.1 $\mathrm{mL}, 18.21 \mathrm{mmol})$ and MOM-Cl $(0.3 \mathrm{~mL}, 4.37 \mathrm{mmol})$ were added sequentially and stirred at $35{ }^{\circ} \mathrm{C}$ temperature for $6 \mathrm{~h}$. The reaction mixture was evaporated and purified the residue by column chromatography (60-120 mesh silica gel, 5\% EtOAc in pet. ether) to afford $32(1.5 \mathrm{~g}$, $76 \%$ ) as a colorless liquid with $100 \%$ diastereoselectivity. $[\alpha]^{25}=+81.1\left(c 0.5, \mathrm{CHCl}_{3}\right)$; IR (neat): $3450,2927,2856,1741,1636,1464,1377,1248,1092,836,773,696 \mathrm{~cm}^{-1} ;{ }^{1} \mathrm{H}$ NMR $\left(\mathrm{CDCl}_{3}, 300 \mathrm{MHz}\right): \delta$ 7.50-7.44 (m, 5H, ArH-Bn), 6.10-6.01 (m, 1H, olefinic), 5.28-5.21 (m, 2H, olefinic), 4.99 (d, 1H, $J=11.3 \mathrm{~Hz}$, benzylic), 4.84 (d, 1H, $J=6.7 \mathrm{~Hz},-\mathrm{OCH}), 4.79$ (d, 
$1 \mathrm{H}, J=6.7 \mathrm{~Hz},-\mathrm{OCH}), 4.74(\mathrm{~d}, 1 \mathrm{H}, J=11.3 \mathrm{~Hz}$, benzylic), 4.30-4.26 (m,1H, -OCH), 3.94$3.83\left(\mathrm{~m}, 3 \mathrm{H},-\mathrm{OCH}_{2},-\mathrm{OCH}\right), 3.70-3.68(\mathrm{~m}, 1 \mathrm{H},-\mathrm{OCH}), 3.53\left(\mathrm{~s}, 3 \mathrm{H},-\mathrm{OCH}_{3}\right), 2.67-2.58(\mathrm{~m}$, $\left.2 \mathrm{H},-\mathrm{CH}_{2}\right), 2.07-1.88\left(\mathrm{~m}, 2 \mathrm{H},-\mathrm{CH}_{2}\right), 1.07-1.04\left(\mathrm{~m}, 18 \mathrm{H}, 6 \mathrm{x}-\mathrm{CH}_{3}\right), 0.24(\mathrm{~d}, 6 \mathrm{H}, J=6.2 \mathrm{~Hz}, 2$ $\left.\mathrm{x}-\mathrm{CH}_{3}\right), 0.09\left(\mathrm{~s}, 6 \mathrm{H}, 2 \mathrm{x}-\mathrm{CH}_{3}\right) ;{ }^{13} \mathrm{C} \mathrm{NMR}\left(\mathrm{CDCl}_{3}, 75 \mathrm{MHz}\right): \delta 138.9,133.3,128.3,127.9$, $127.3,117.9,83.8,80.0,74.4,73.3,69.7,59.1,36.2,35.4,25.9,21.1,17.9,-3.6,-4.9,-5.3$; HRMS (ESI): $m / z$ calculated for $\mathrm{C}_{29} \mathrm{H}_{55} \mathrm{O}_{5} \mathrm{Si}_{2}[\mathrm{M}+\mathrm{H}]^{+}$539.2425, found 539.2418.

\section{(3R,4R,5S)-4-(Benzyloxy)-3-(tert.-butyldimethylsilyloxy)-5-(methoxymethoxy)oct-7-en-1-} ol (33):

To a cooled $\left(0{ }^{\circ} \mathrm{C}\right)$ solution of $32(1.3 \mathrm{~g}, 2.41 \mathrm{mmol})$ in $\mathrm{MeOH}(15 \mathrm{~mL})$, catalytic amount of PPTS was added and stirred at $35^{\circ} \mathrm{C}$ temperature for $1 \mathrm{~h}$. The reaction mixture was quenched with sat. $\mathrm{NaHCO}_{3}$ solution $(10 \mathrm{~mL})$ and $\mathrm{MeOH}$ was evaporated. $\mathrm{H}_{2} \mathrm{O}(10 \mathrm{~mL})$ was added and the mixture was extracted with EtOAc $(2 \times 10 \mathrm{~mL})$. The organic extracts were washed with brine $(15 \mathrm{~mL})$, dried $\left(\mathrm{Na}_{2} \mathrm{SO}_{4}\right)$ and evaporated. The residue was purified by column chromatography (60-120 mesh silica gel, 13\% EtOAc in pet. ether) to give 33 (0.8 g, $78 \%$ ) as a yellow liquid with $100 \%$ diastereoselectivity. $[\alpha]^{25}=+85.8\left(c\right.$ 0.5, $\left.\mathrm{CHCl}_{3}\right)$; IR (neat): 3031, 2954, 2930, 2886, 2856, 1612, 1585, 1513, 1466, 1387, 1361, 1301, 1094, 1040, 937, 776, $698 \mathrm{~cm}^{-1} ;{ }^{1} \mathrm{H}$ NMR $\left(\mathrm{CDCl}_{3}, 300 \mathrm{MHz}\right): \delta$ 7.39-7.28 (m, 5H, ArH-Bn), 5.975.83 (m, 1H, olefinic), 5.19-5.09 (m, 2H, olefinic), 4.91 (d, 1H, J=11.1 Hz, benzylic), 4.68 (s, 2H, $\left.-\mathrm{OCH}_{2}\right), 4.67$ (d, 1H, J=10.9 Hz, benzylic), 4.26-4.18 (m, 1H, -OCH), 3.87-3.69 (m, $\left.3 \mathrm{H},-\mathrm{OCH}_{2},-\mathrm{OCH}\right), 3.64-3.59(\mathrm{~m}, 1 \mathrm{H},-\mathrm{OCH}), 3.40\left(\mathrm{~s}, 3 \mathrm{H},-\mathrm{OCH}_{3}\right), 2.67-2.28\left(\mathrm{~m}, 2 \mathrm{H},-\mathrm{CH}_{2}\right)$, 2.06-1.81 (m, 2H, $\left.-\mathrm{CH}_{2}\right), 0.96\left(\mathrm{~s}, 9 \mathrm{H}, 3 \mathrm{x}-\mathrm{CH}_{3}\right), 0.14\left(\mathrm{~s}, 6 \mathrm{H}, 2 \mathrm{x}-\mathrm{CH}_{3}\right) ;{ }^{13} \mathrm{C} \mathrm{NMR}\left(\mathrm{CDCl}_{3}, 75\right.$ $\mathrm{MHz}): \delta 138.3,134.6,128.3,127.8,127.6,117.4,96.2,83.4,77.2,74.4,71.1,59.2,55.9$, 35.2, 34.7, 25.8, 17.9, -4.3, -4.7; HRMS (ESI): $\mathrm{m} / \mathrm{z}$ calculated for $\mathrm{C}_{23} \mathrm{H}_{40} \mathrm{O}_{5} \mathrm{NaSi}[\mathrm{M}+\mathrm{Na}]^{+}$ 447.1589, found 447.1592 . 
(3R,4R,5S)-4-(Benzyloxy)-3-(tert.-butyldimethylsilyloxy)-5-(methoxymethoxy)oct-7enoic acid (7):

To a stirred solution of $\mathbf{3 3}(0.6 \mathrm{~g}, 1.41 \mathrm{mmol})$ in $\mathrm{CH}_{2} \mathrm{Cl}_{2}: \mathrm{H}_{2} \mathrm{O}(1: 1,13 \mathrm{~mL})$ TEMPO (0.06 g, $0.42 \mathrm{mmol})$ and BAIB (1.36 g, $4.24 \mathrm{mmol})$ were added at $0{ }^{\circ} \mathrm{C}$ and stirred for $2 \mathrm{~h}$. Work up as described for 5 and purification of the residue by column chromatography (60120 mesh silica gel, $20-24 \%$ EtOAc in pet. ether) to give acid $7(0.45 \mathrm{~g}, 72 \%)$ as a colorless gummy oil with $100 \%$ diastereoselectivity. $[\alpha]^{25}{ }_{\mathrm{D}}=+54.4\left(\right.$ c $\left.0.5, \mathrm{CHCl}_{3}\right)$; IR (neat): 3460 , $3161,2931,2858,1712,1641,1397,1257,1098,917,771,699 \mathrm{~cm}^{-1} ;{ }^{1} \mathrm{H}$ NMR $\left(\mathrm{CDCl}_{3}, 300\right.$ MHz): $\delta$ 7.36-7.27 (m, 5H, ArH-Bn), 5.95-5.79 (m, 1H, olefinic), 5.15-5.07 (m, 2H, olefinic), $4.84\left(\mathrm{~d}, 1 \mathrm{H}, J=11.3 \mathrm{~Hz}\right.$, benzylic), $4.66\left(\mathrm{~s}, 2 \mathrm{H},-\mathrm{OCH}_{2}\right), 4.63(\mathrm{~d}, 1 \mathrm{H}, J=11.3 \mathrm{~Hz}$, benzylic), 4.48-4.42 (m, 1H, -OCH), 3.79-3.72 (m, 1H, -OCH), 3.59-3.55 (m, 1H, -OCH), 3.39 (s, 3H, $\left.\mathrm{OCH}_{3}\right), 2.69\left(\mathrm{~d}, 2 \mathrm{H}, J=5.6 \mathrm{~Hz},-\mathrm{CH}_{2}\right), 2.48-2.41\left(\mathrm{~m}, 2 \mathrm{H},-\mathrm{CH}_{2}\right), 0.89\left(\mathrm{~s}, 9 \mathrm{H}, 3 \mathrm{x}-\mathrm{CH}_{3}\right), 0.10$ $\left(\mathrm{d}, 6 \mathrm{H}, J=6.6 \mathrm{~Hz}, 2 \mathrm{x}-\mathrm{CH}_{3}\right) ;{ }^{13} \mathrm{C} \mathrm{NMR}\left(\mathrm{CDCl}_{3}, 75 \mathrm{MHz}\right): \delta 176.9,138.3,137.4,134.5$, $130.2,128.3,127.7,127.6,117.5,95.9,83.3,76.5,73.9,69.9,55.9,38.3,35.1,25.7,17.9$, 4.4, -4.9; HRMS (ESI): $\mathrm{m} / \mathrm{z}$ calculated for $\mathrm{C}_{23} \mathrm{H}_{38} \mathrm{O}_{6} \mathrm{NaSi}[\mathrm{M}+\mathrm{Na}]^{+}$461.3348, found 461.3359 . 


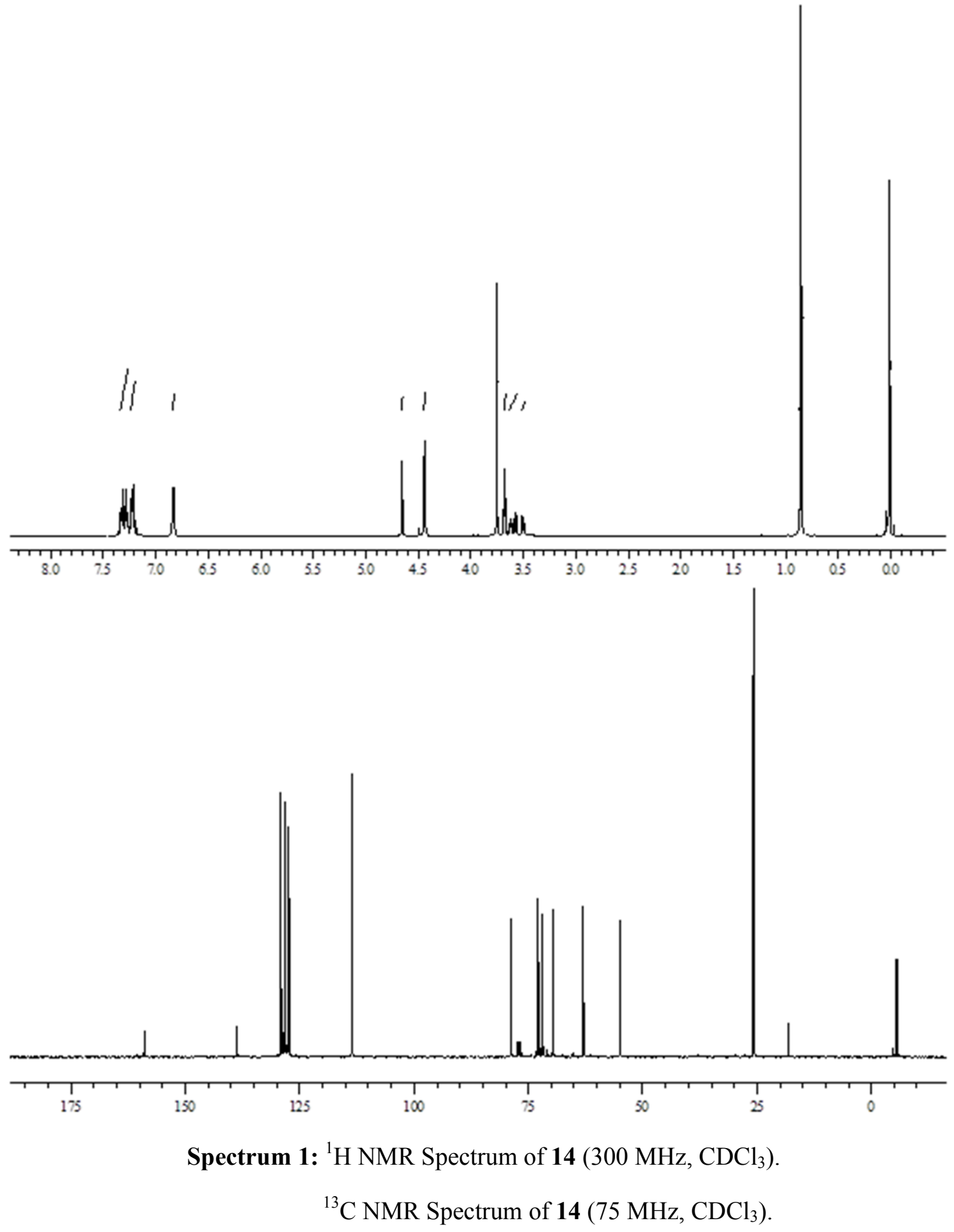



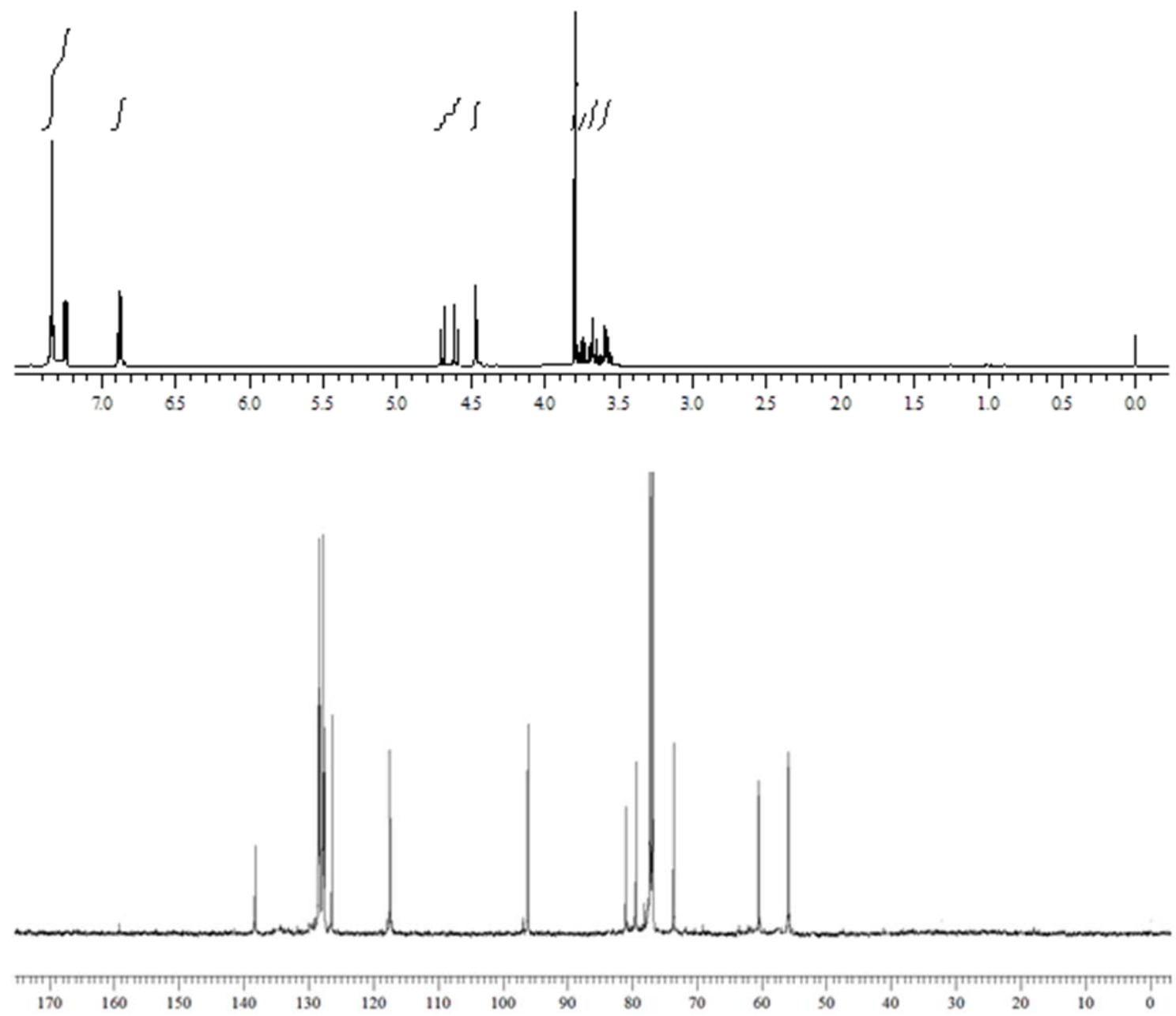

Spectrum 2: ${ }^{1} \mathrm{H}$ NMR Spectrum of $15\left(300 \mathrm{MHz}, \mathrm{CDCl}_{3}\right)$.

${ }^{13} \mathrm{C}$ NMR Spectrum of $15\left(75 \mathrm{MHz}, \mathrm{CDCl}_{3}\right)$. 

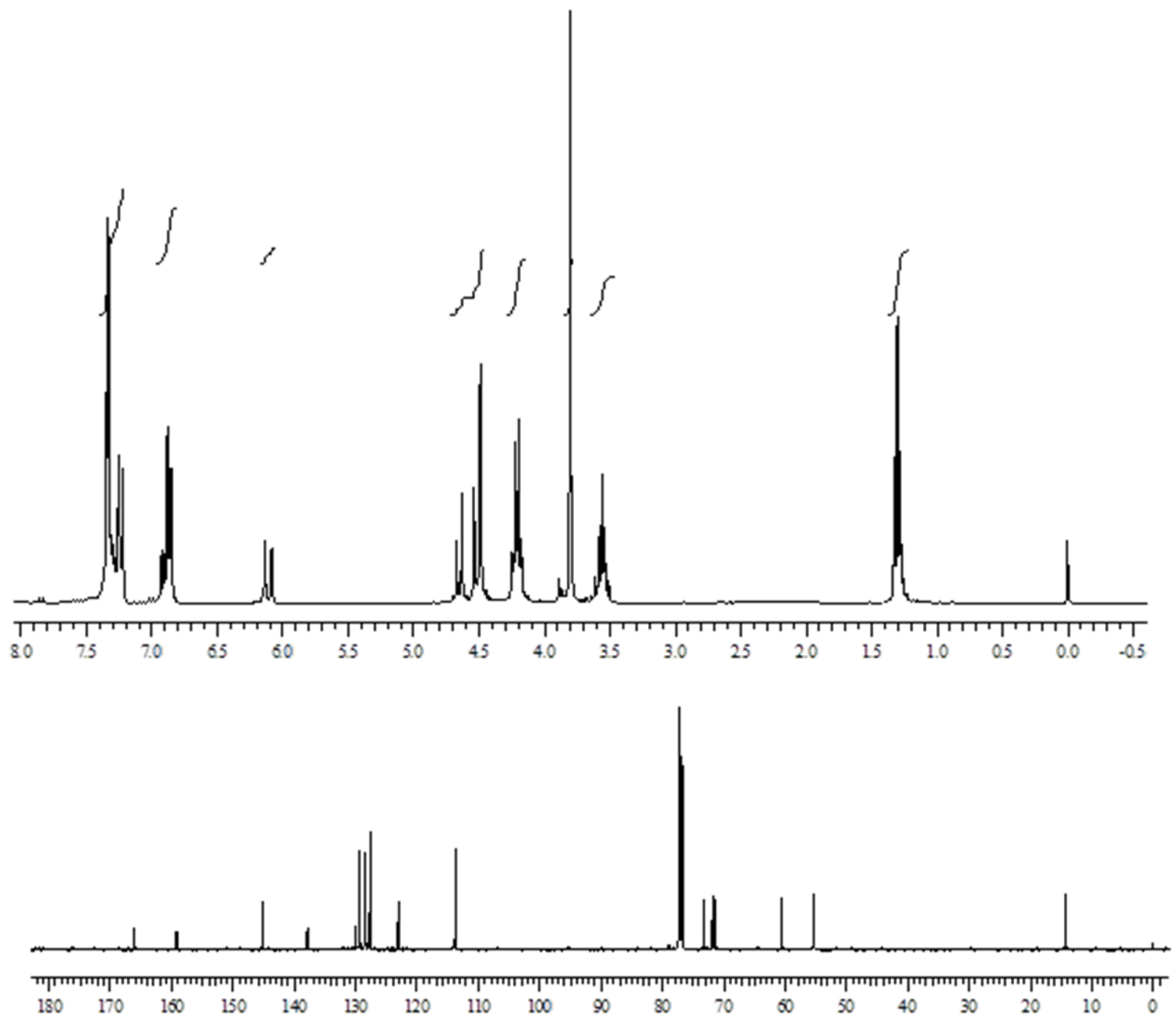

Spectrum 3: ${ }^{1} \mathrm{H}$ NMR Spectrum of $\mathbf{1 6}\left(300 \mathrm{MHz}, \mathrm{CDCl}_{3}\right)$.

${ }^{13} \mathrm{C}$ NMR Spectrum of $16\left(75 \mathrm{MHz}, \mathrm{CDCl}_{3}\right)$. 

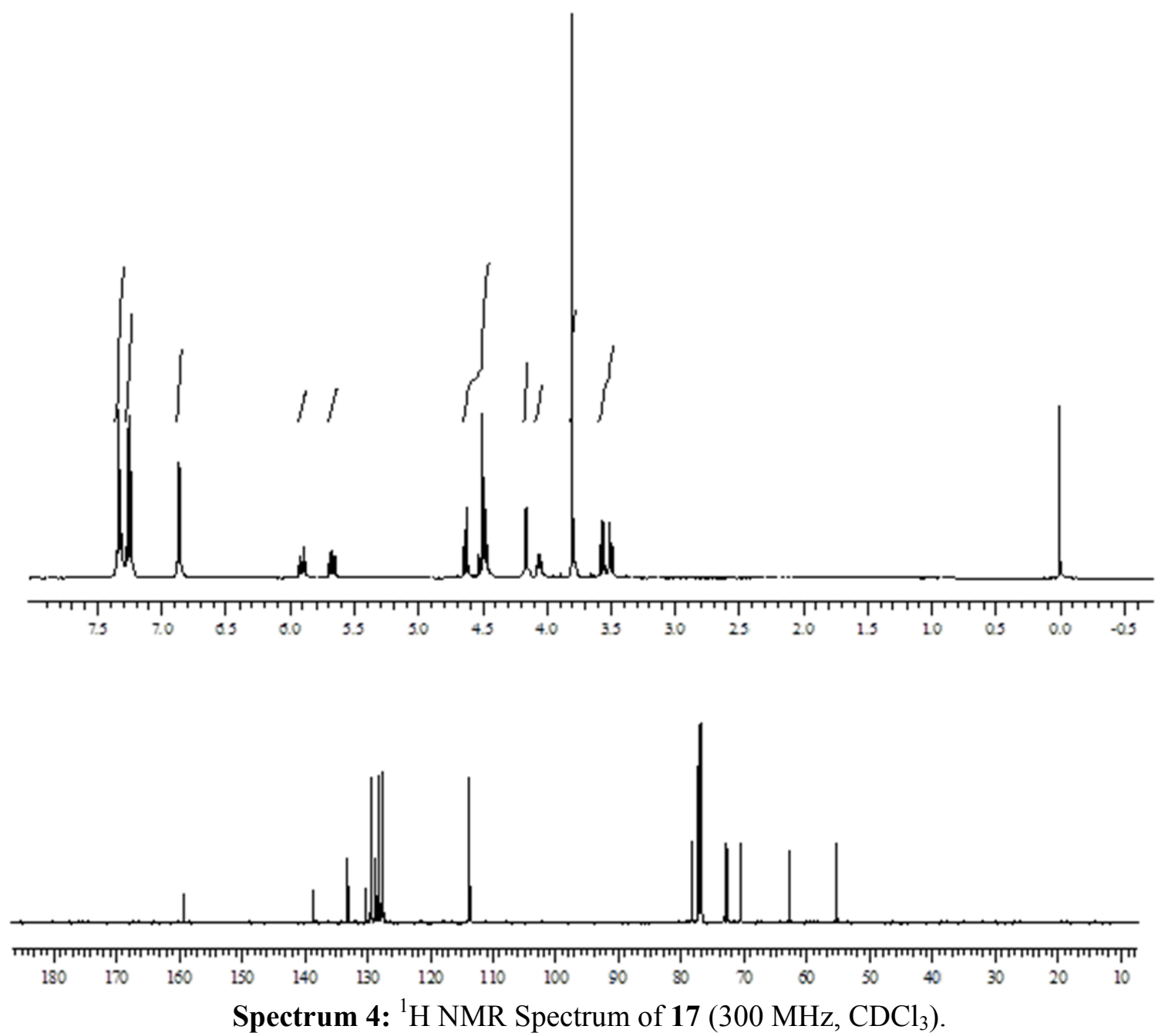

${ }^{13} \mathrm{C}$ NMR Spectrum of $17\left(75 \mathrm{MHz}, \mathrm{CDCl}_{3}\right)$. 

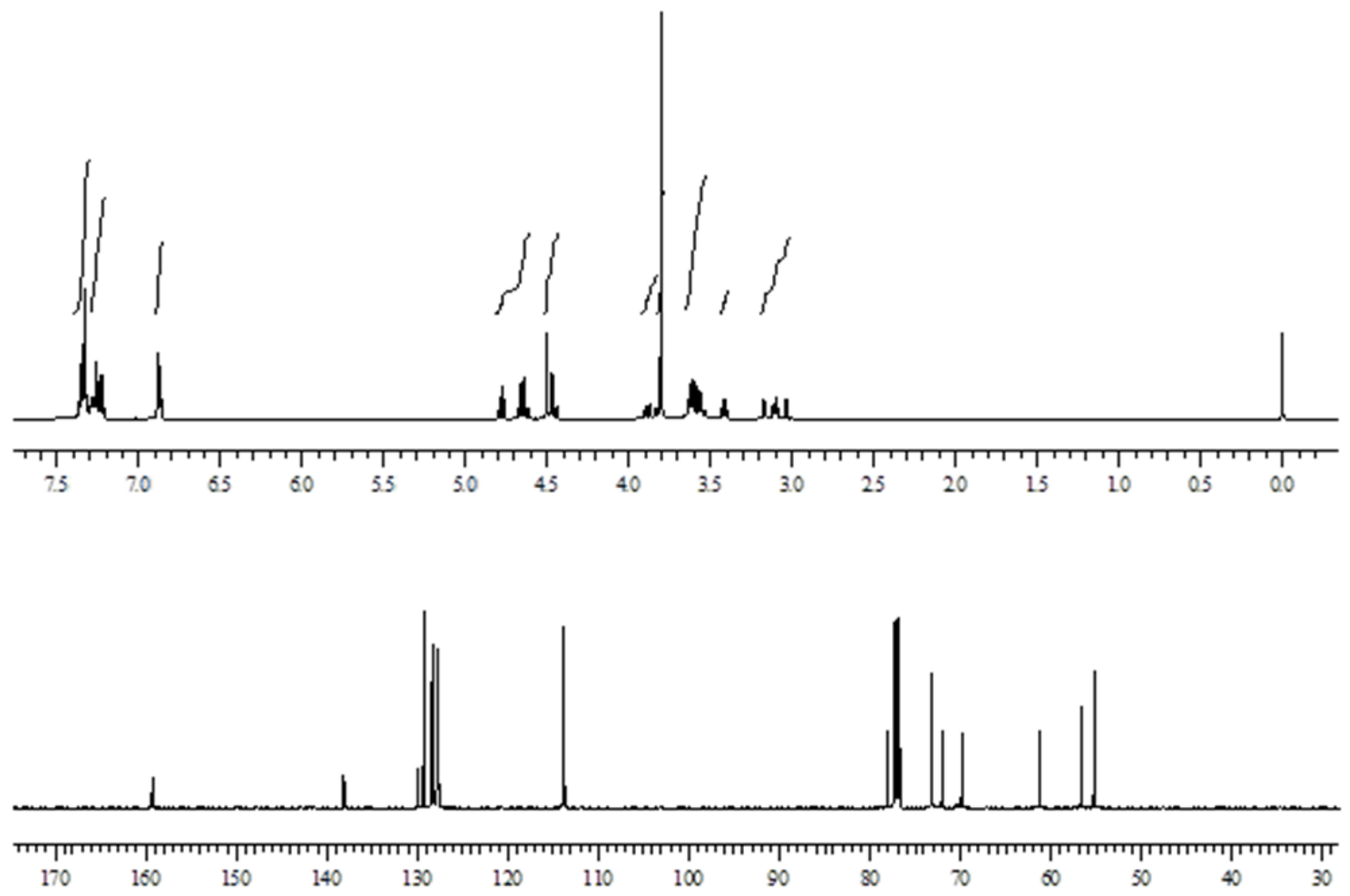

Spectrum 5: ${ }^{1} \mathrm{H}$ NMR Spectrum of $18\left(300 \mathrm{MHz}, \mathrm{CDCl}_{3}\right)$.

${ }^{13} \mathrm{C}$ NMR Spectrum of $18\left(75 \mathrm{MHz}, \mathrm{CDCl}_{3}\right)$. 


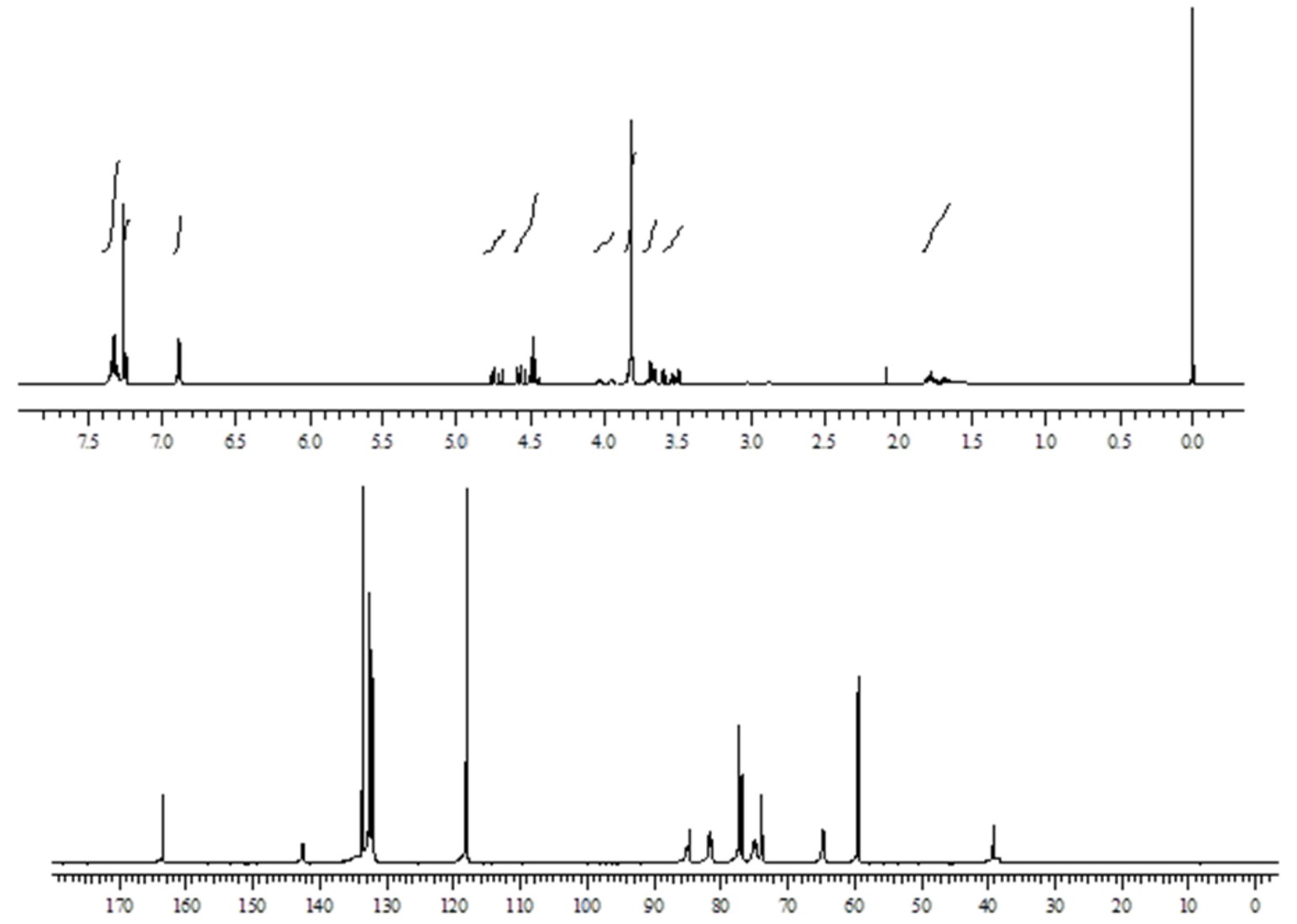

Spectrum 6: ${ }^{1} \mathrm{H}$ NMR Spectrum of $19\left(300 \mathrm{MHz}, \mathrm{CDCl}_{3}\right)$.

${ }^{13} \mathrm{C}$ NMR Spectrum of $19\left(75 \mathrm{MHz}, \mathrm{CDCl}_{3}\right)$. 

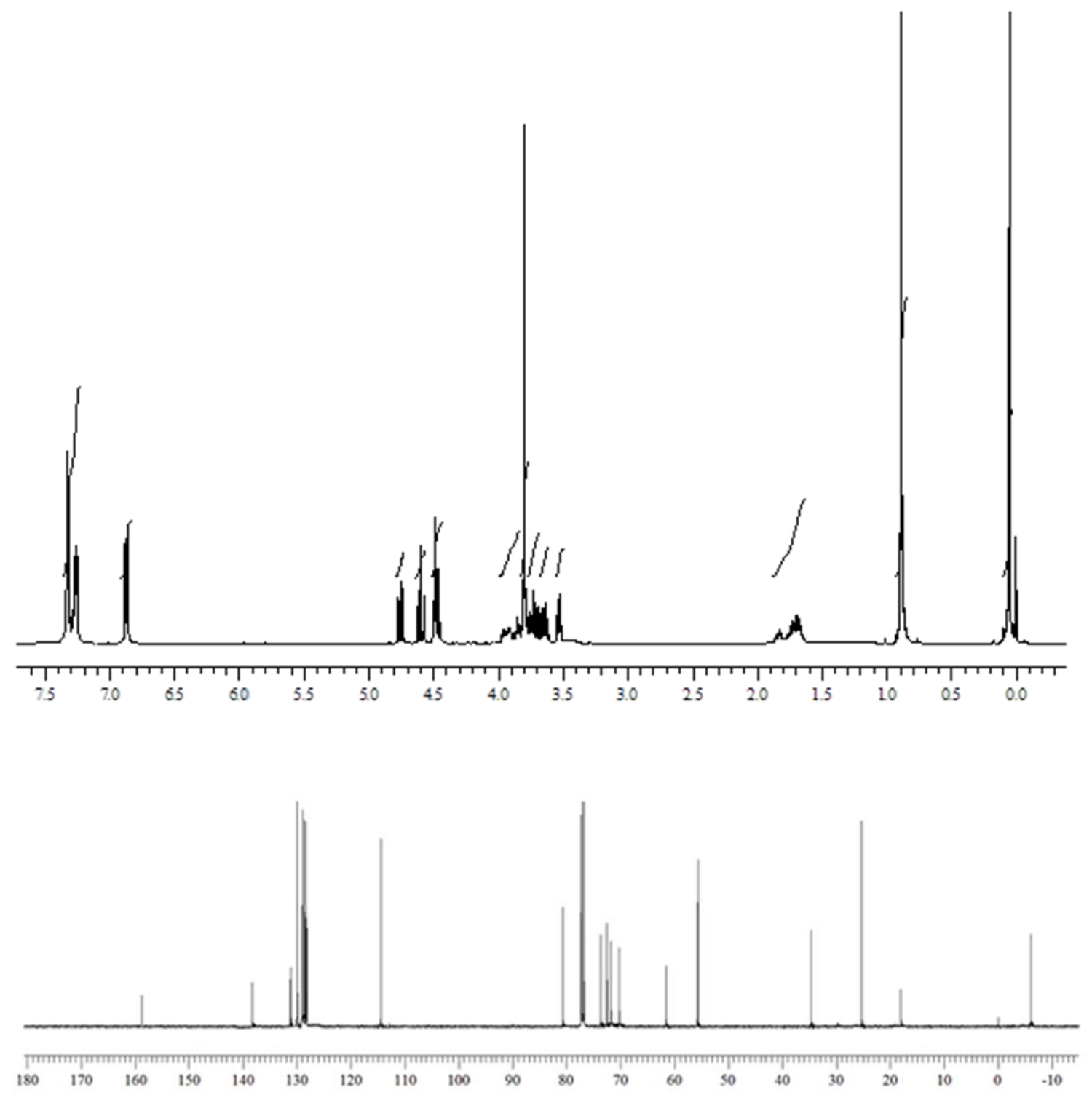

Spectrum 7: ${ }^{1} \mathrm{H}$ NMR Spectrum of $11\left(300 \mathrm{MHz}, \mathrm{CDCl}_{3}\right)$.

${ }^{13} \mathrm{C}$ NMR Spectrum of 11 (75 MHz, $\left.\mathrm{CDCl}_{3}\right)$. 


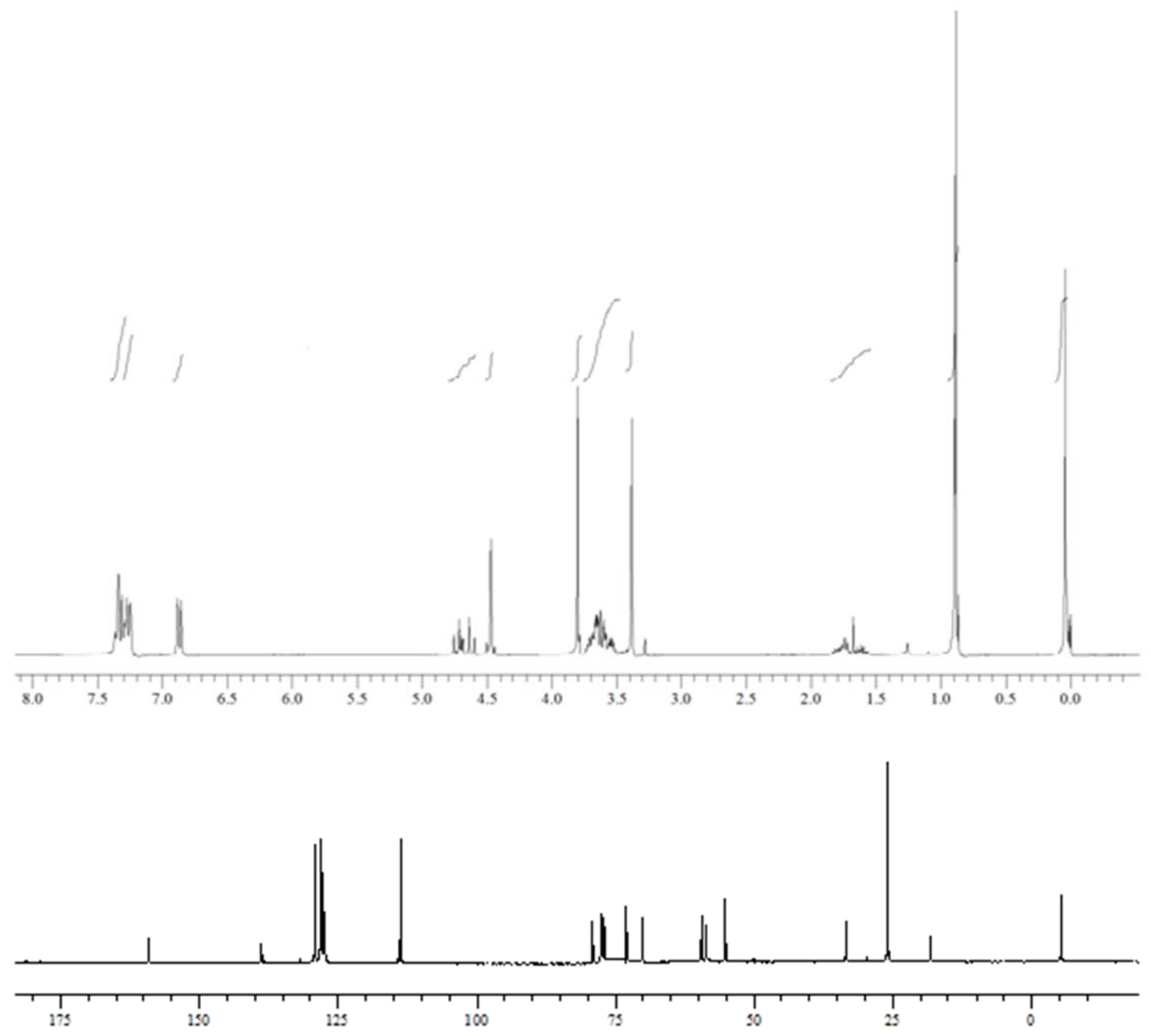

Spectrum 8: ${ }^{1} \mathrm{H}$ NMR Spectrum of $9\left(300 \mathrm{MHz}, \mathrm{CDCl}_{3}\right)$.

${ }^{13} \mathrm{C}$ NMR Spectrum of $9\left(75 \mathrm{MHz}, \mathrm{CDCl}_{3}\right)$. 

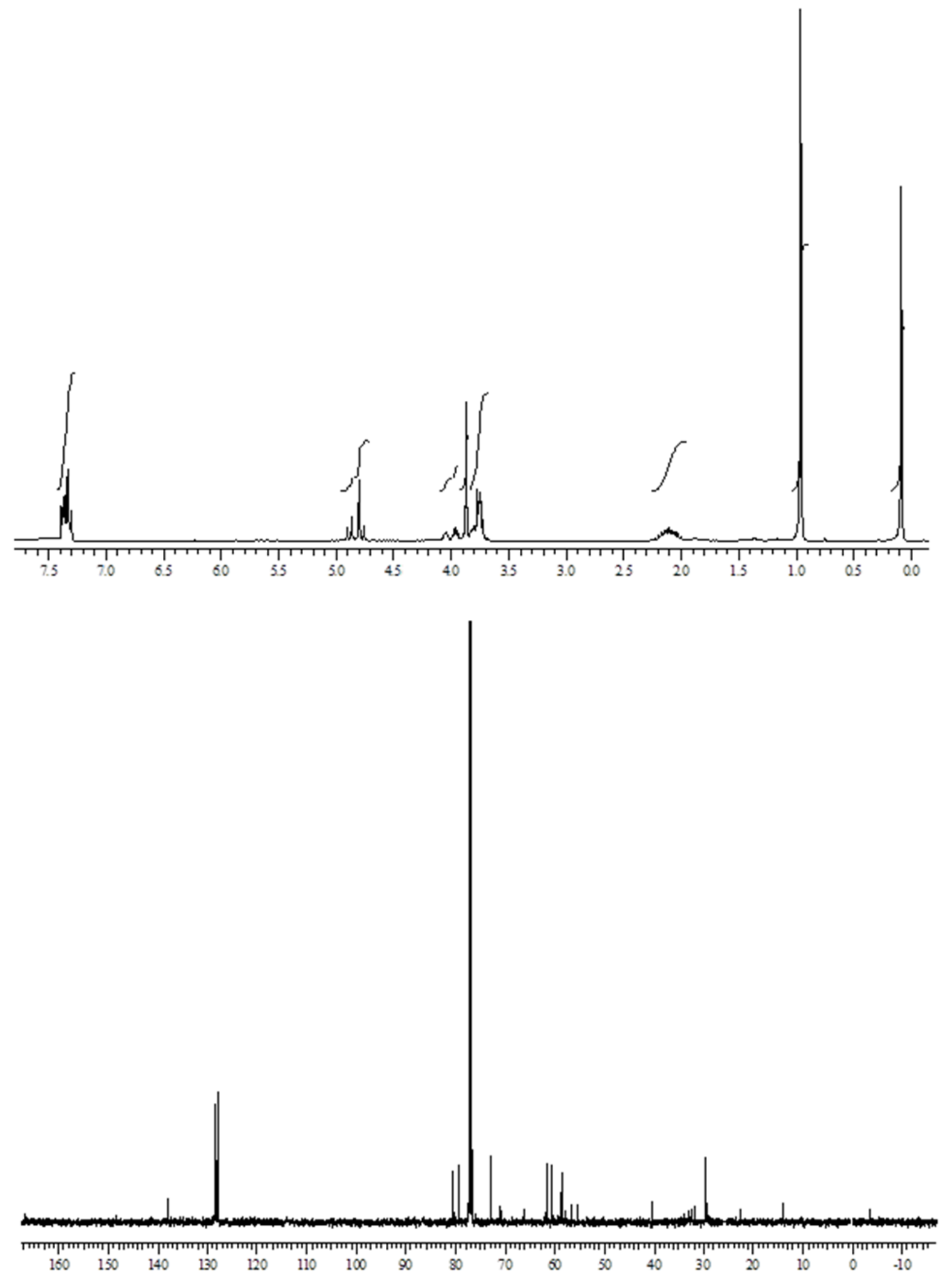

Spectrum 9: ${ }^{1} \mathrm{H}$ NMR Spectrum of $20\left(300 \mathrm{MHz}, \mathrm{CDCl}_{3}\right)$.

${ }^{13} \mathrm{C}$ NMR Spectrum of $20\left(75 \mathrm{MHz}, \mathrm{CDCl}_{3}\right)$. 

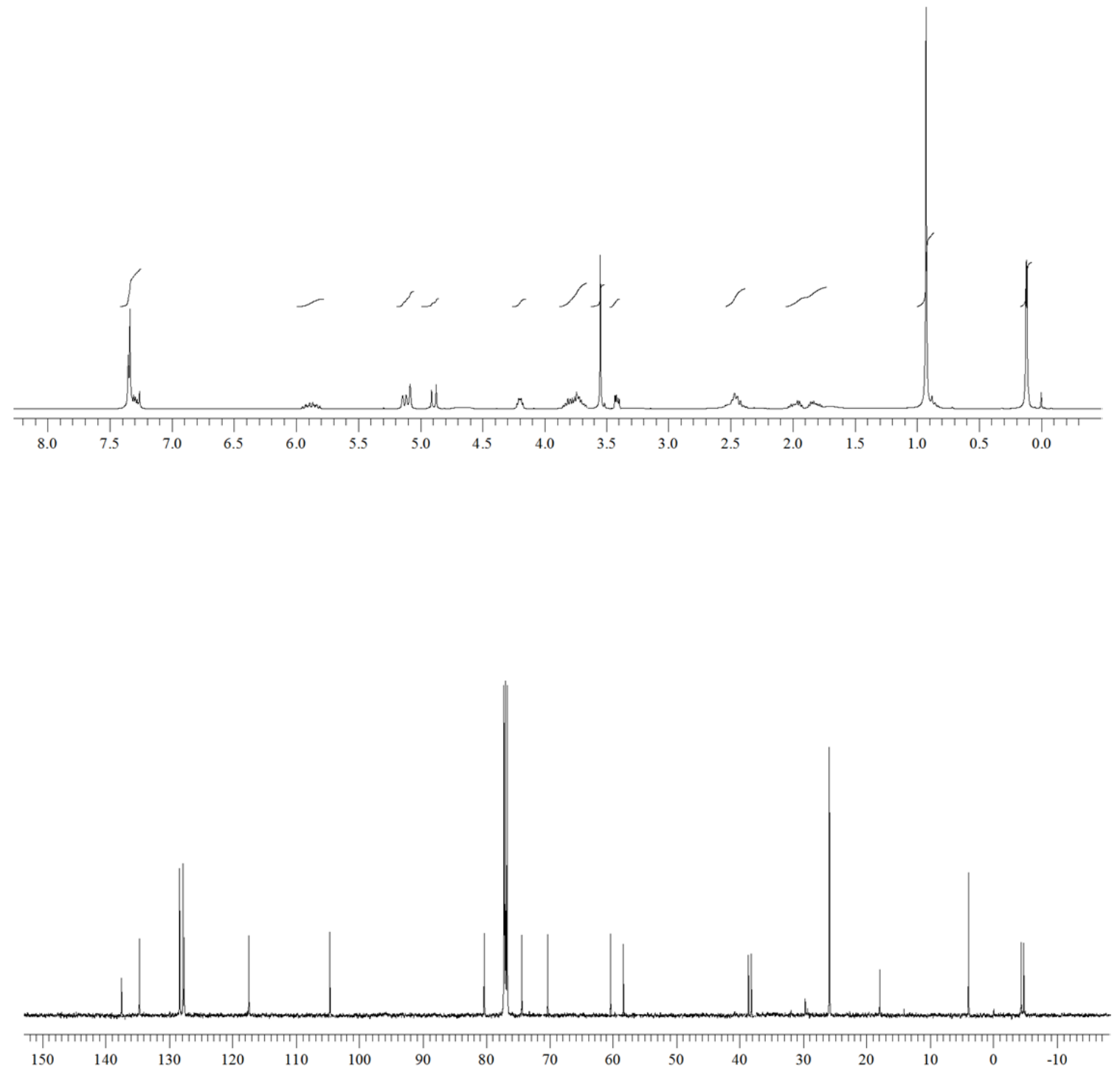

Spectrum 10: ${ }^{1} \mathrm{H}$ NMR Spectrum of $21\left(300 \mathrm{MHz}, \mathrm{CDCl}_{3}\right)$.

${ }^{13} \mathrm{C}$ NMR Spectrum of $21\left(75 \mathrm{MHz}, \mathrm{CDCl}_{3}\right)$. 

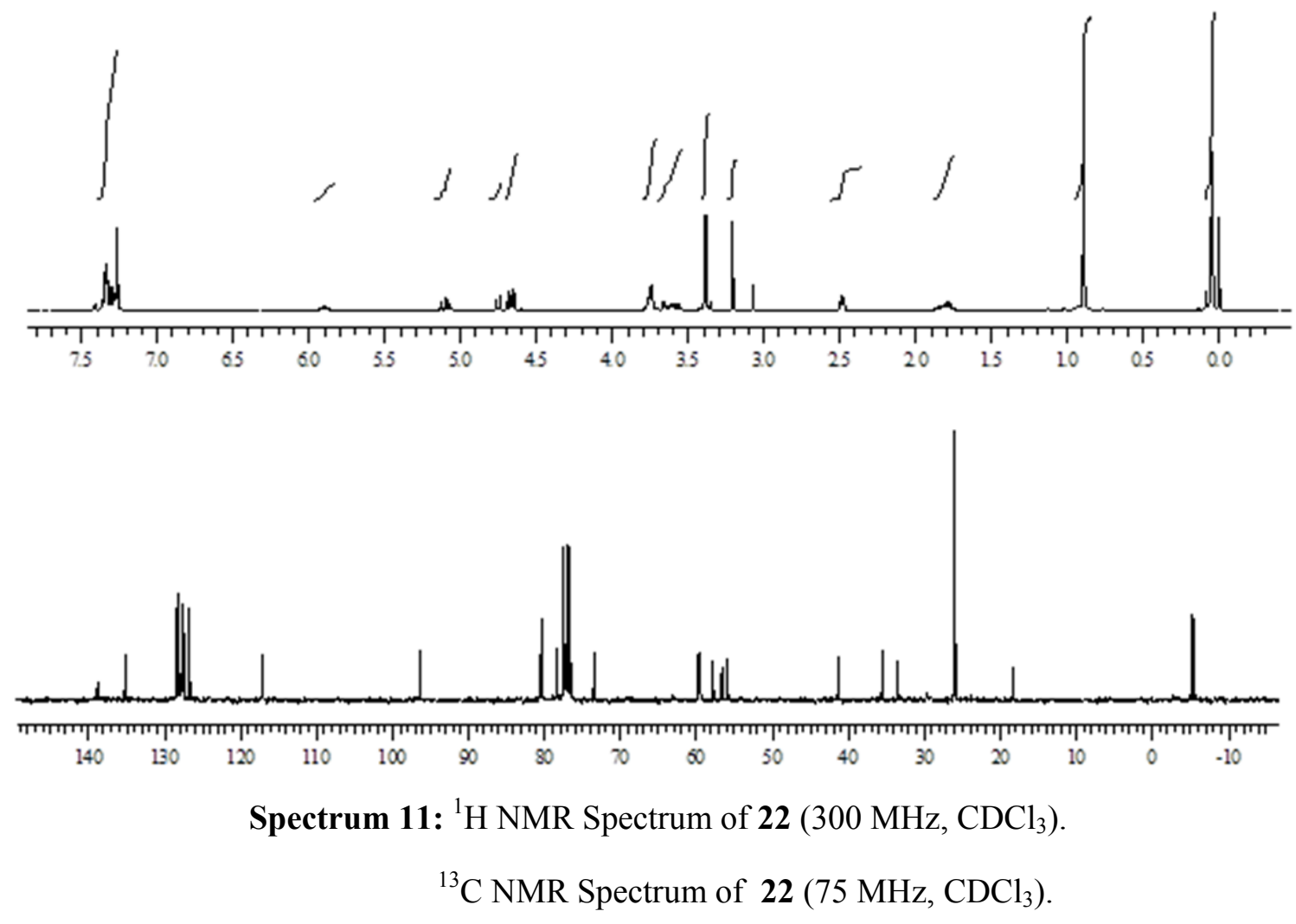

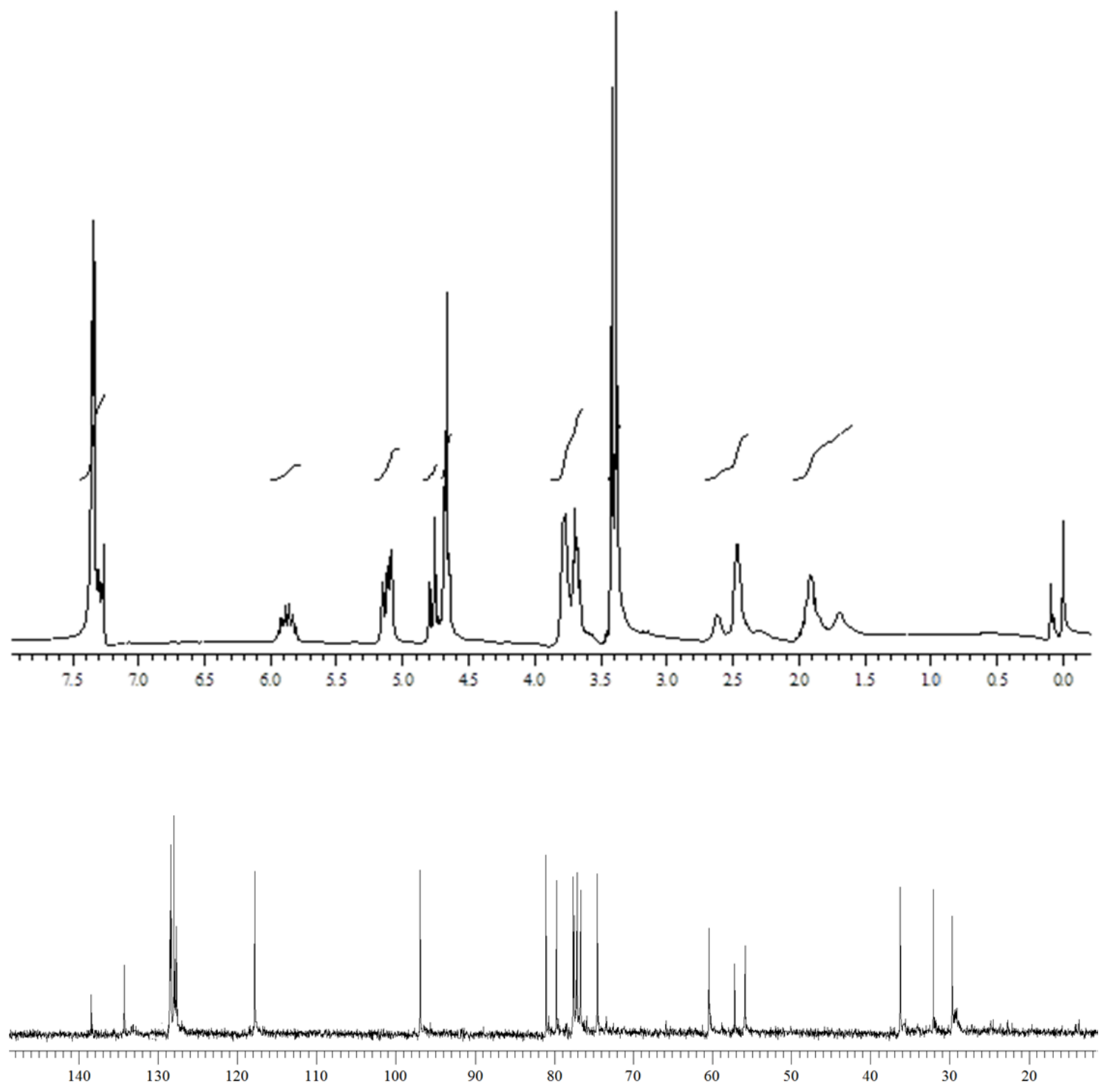

Spectrum 12: ${ }^{1} \mathrm{H}$ NMR Spectrum of $23\left(300 \mathrm{MHz}, \mathrm{CDCl}_{3}\right)$.

${ }^{13} \mathrm{C}$ NMR Spectrum of $23\left(75 \mathrm{MHz}, \mathrm{CDCl}_{3}\right)$. 

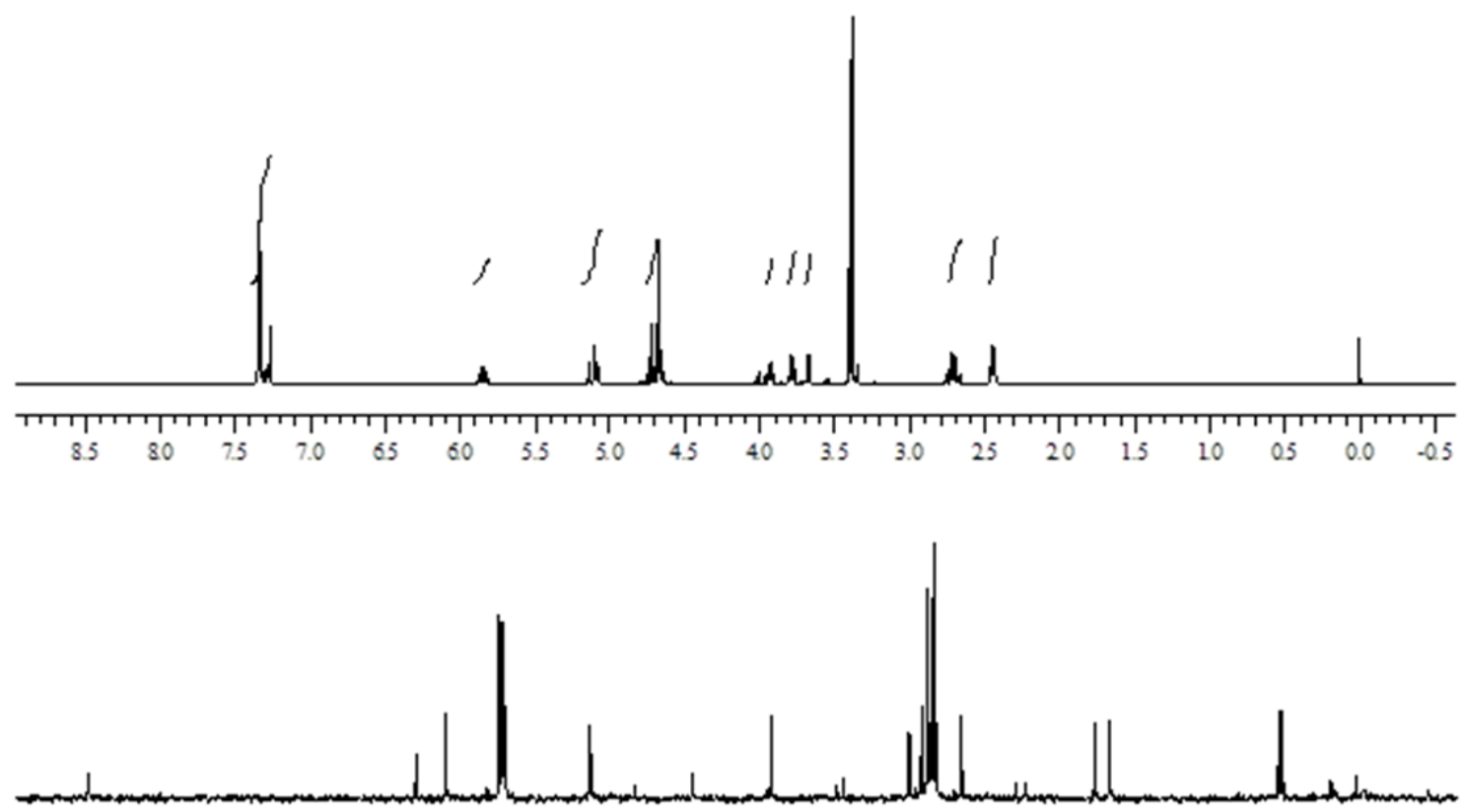

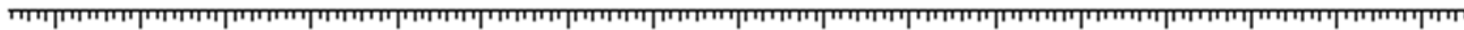

Spectrum 13: ${ }^{1} \mathrm{H}$ NMR Spectrum of $5\left(300 \mathrm{MHz}, \mathrm{CDCl}_{3}\right)$.

${ }^{13} \mathrm{C}$ NMR Spectrum of $\mathbf{5}\left(75 \mathrm{MHz}, \mathrm{CDCl}_{3}\right)$. 

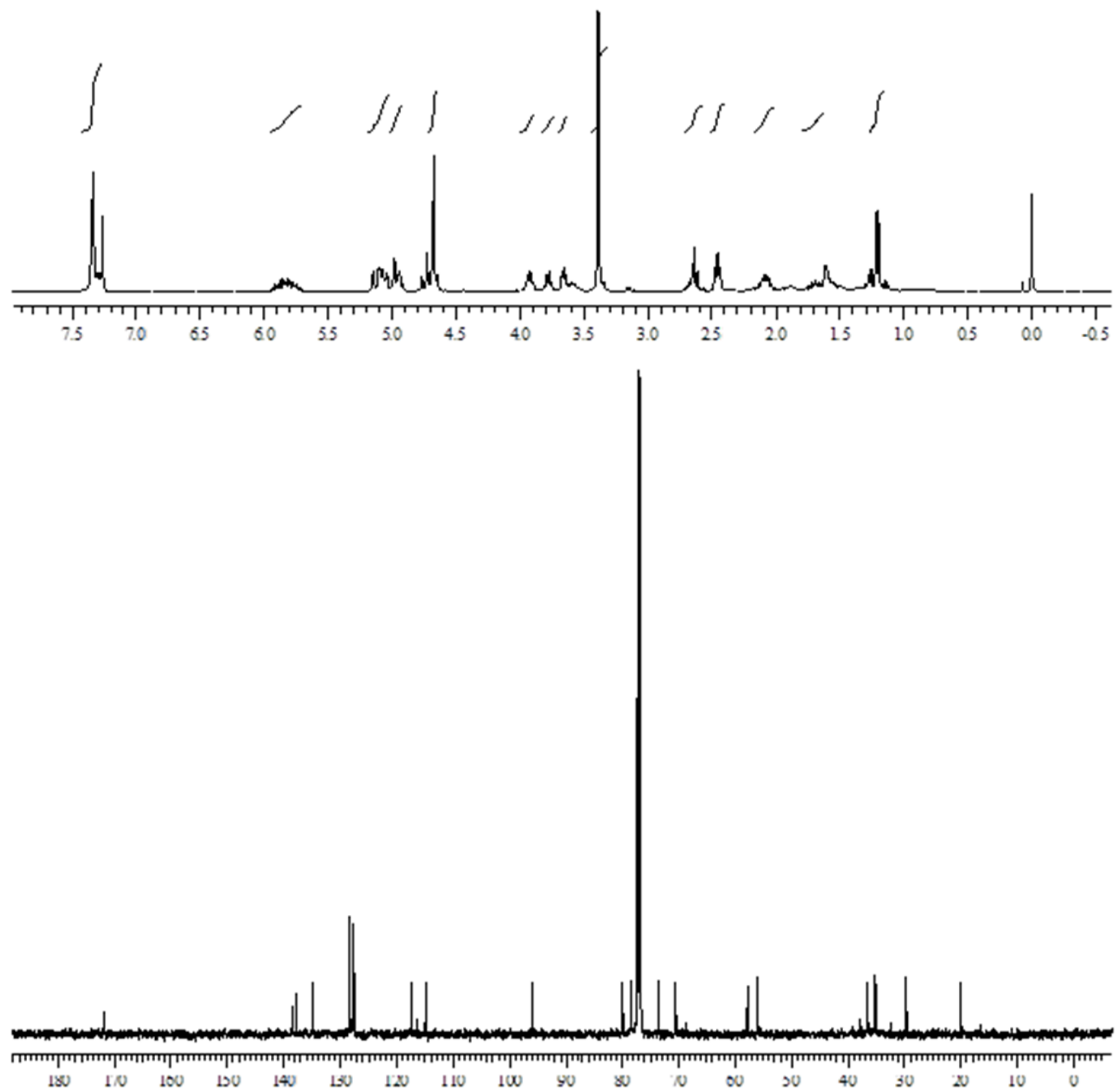

Spectrum 14: ${ }^{1} \mathrm{H}$ NMR Spectrum of $3\left(300 \mathrm{MHz}, \mathrm{CDCl}_{3}\right)$.

${ }^{13} \mathrm{C}$ NMR Spectrum of $3\left(75 \mathrm{MHz}, \mathrm{CDCl}_{3}\right)$. 

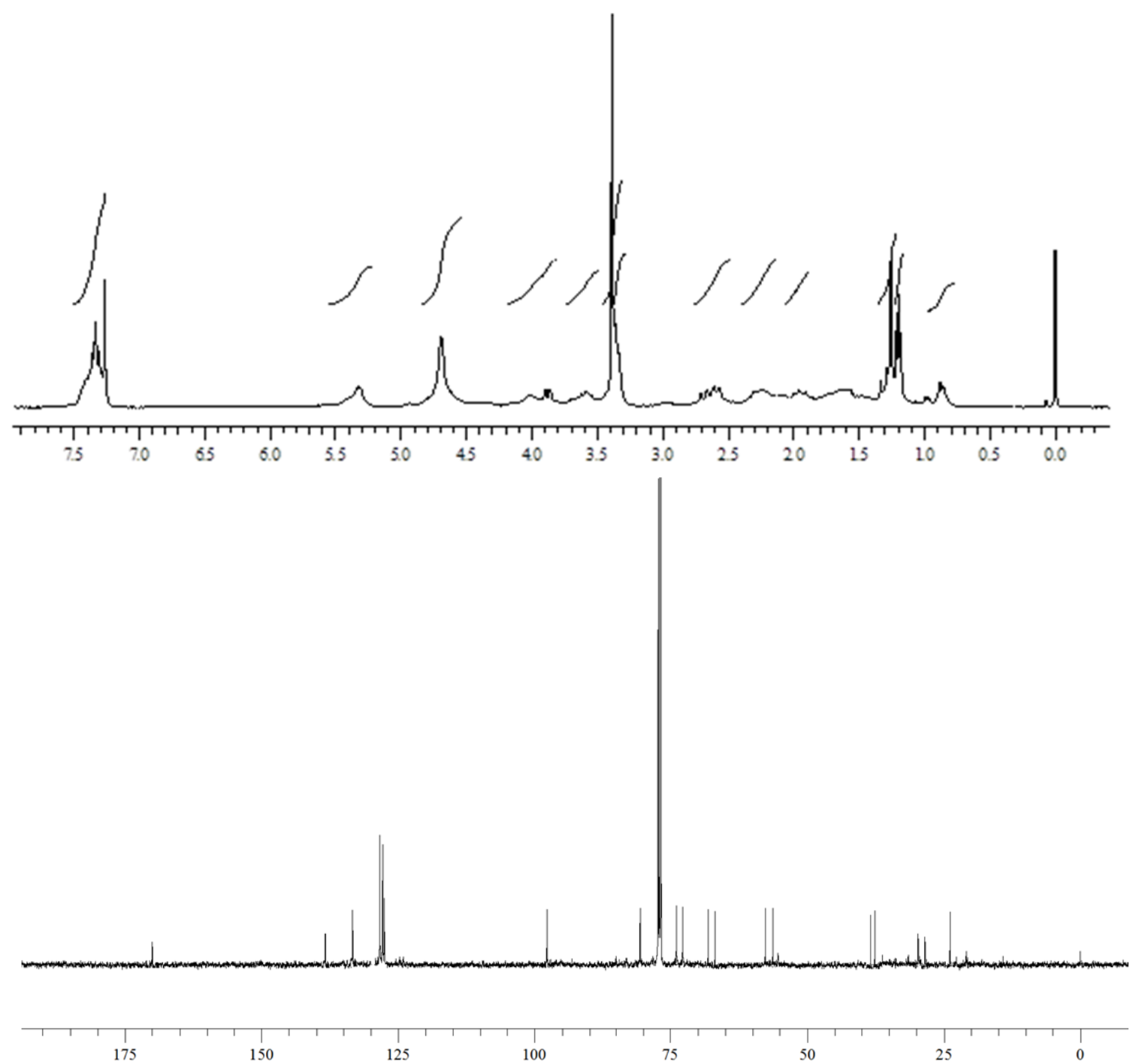

Spectrum 15: ${ }^{1} \mathrm{H}$ NMR Spectrum of $24\left(300 \mathrm{MHz}, \mathrm{CDCl}_{3}\right)$.

${ }^{13} \mathrm{C}$ NMR Spectrum of $24\left(75 \mathrm{MHz}, \mathrm{CDCl}_{3}\right)$. 


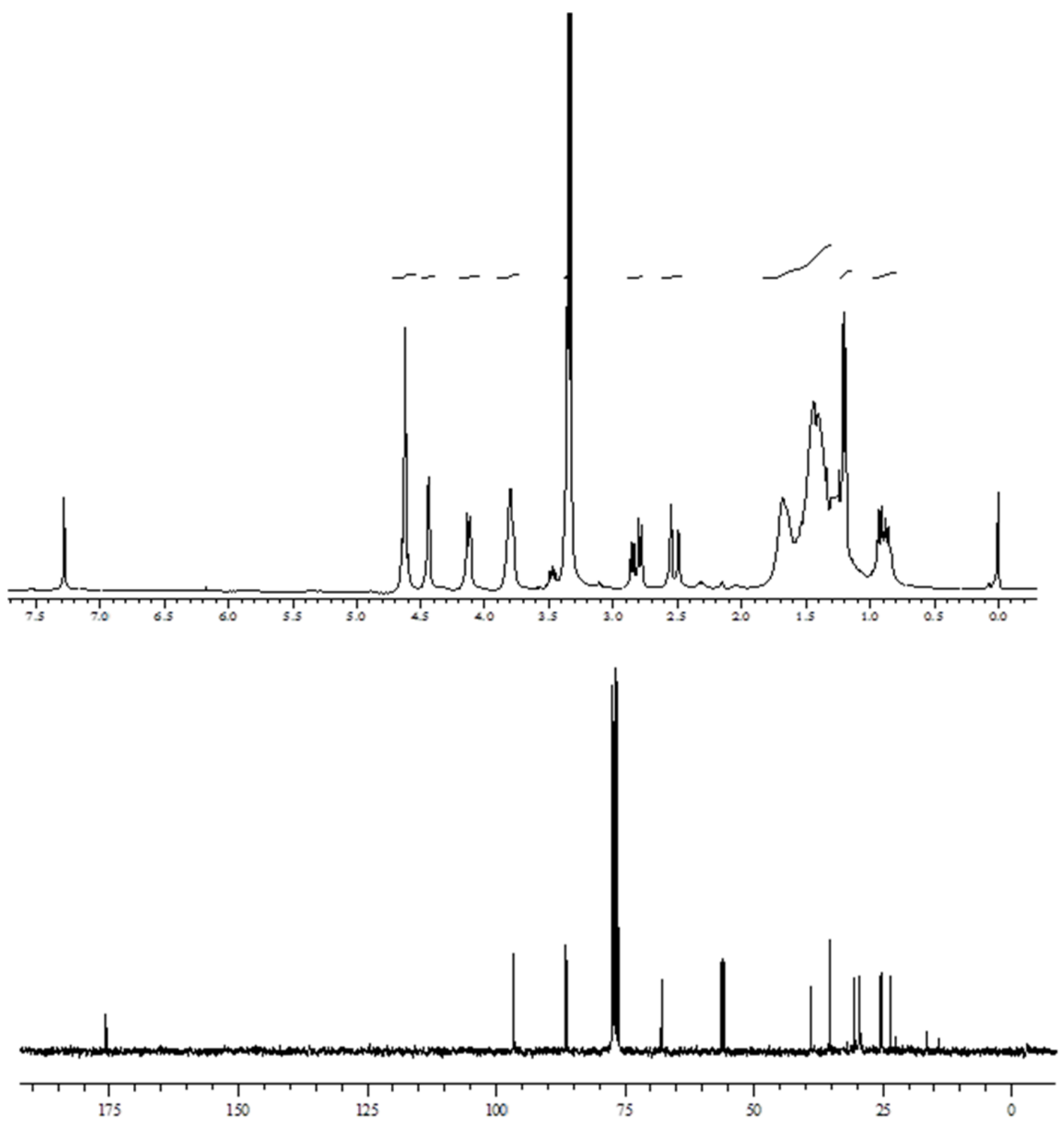

Spectrum 16: ${ }^{1} \mathrm{H}$ NMR Spectrum of $25\left(300 \mathrm{MHz}, \mathrm{CDCl}_{3}\right)$.

${ }^{13} \mathrm{C}$ NMR Spectrum of $25\left(75 \mathrm{MHz}, \mathrm{CDCl}_{3}\right)$. 

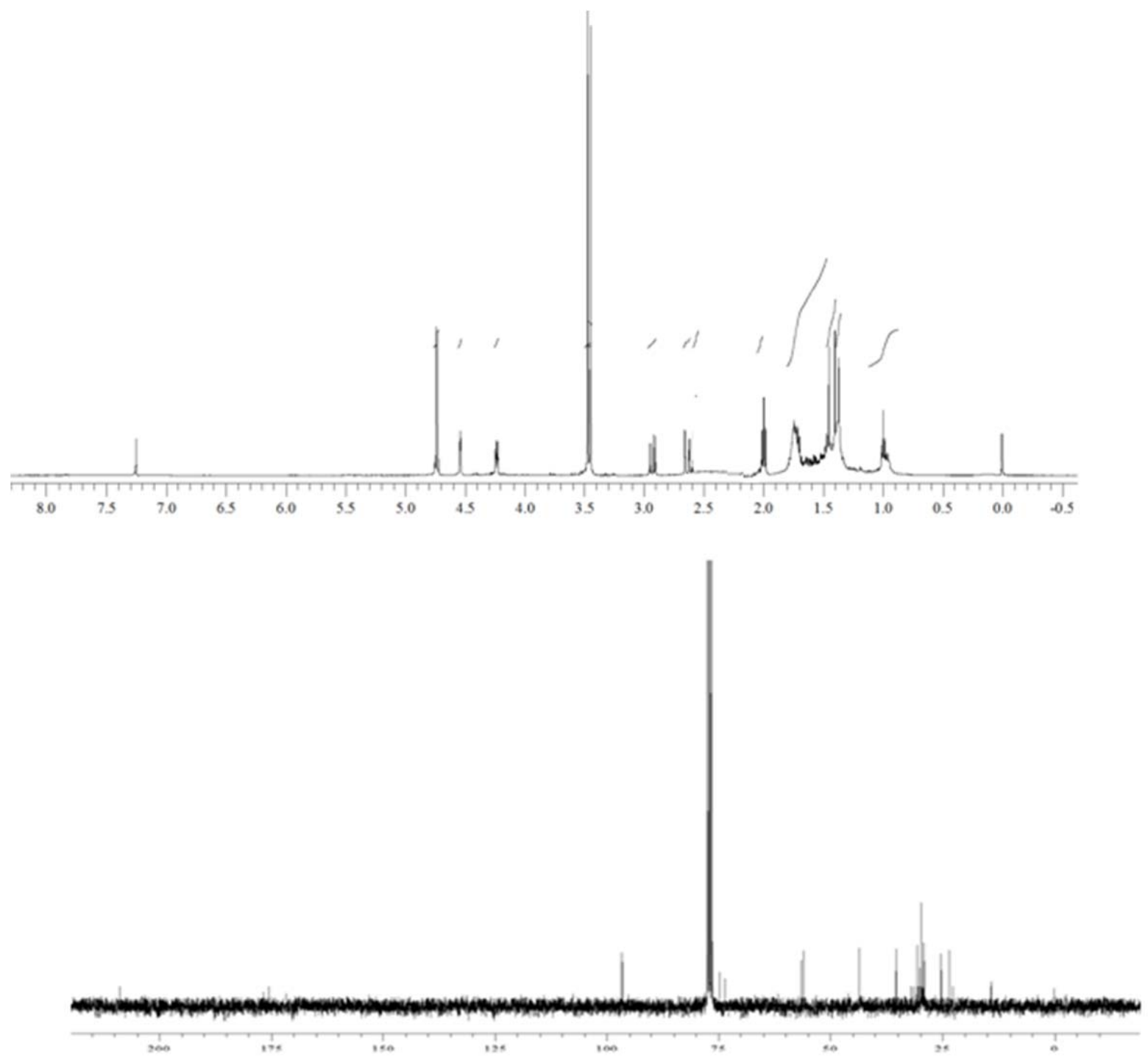

Spectrum 17: ${ }^{1} \mathrm{H}$ NMR Spectrum of $26\left(300 \mathrm{MHz}, \mathrm{CDCl}_{3}\right)$.

${ }^{13} \mathrm{C}$ NMR Spectrum of $26\left(75 \mathrm{MHz}, \mathrm{CDCl}_{3}\right)$. 

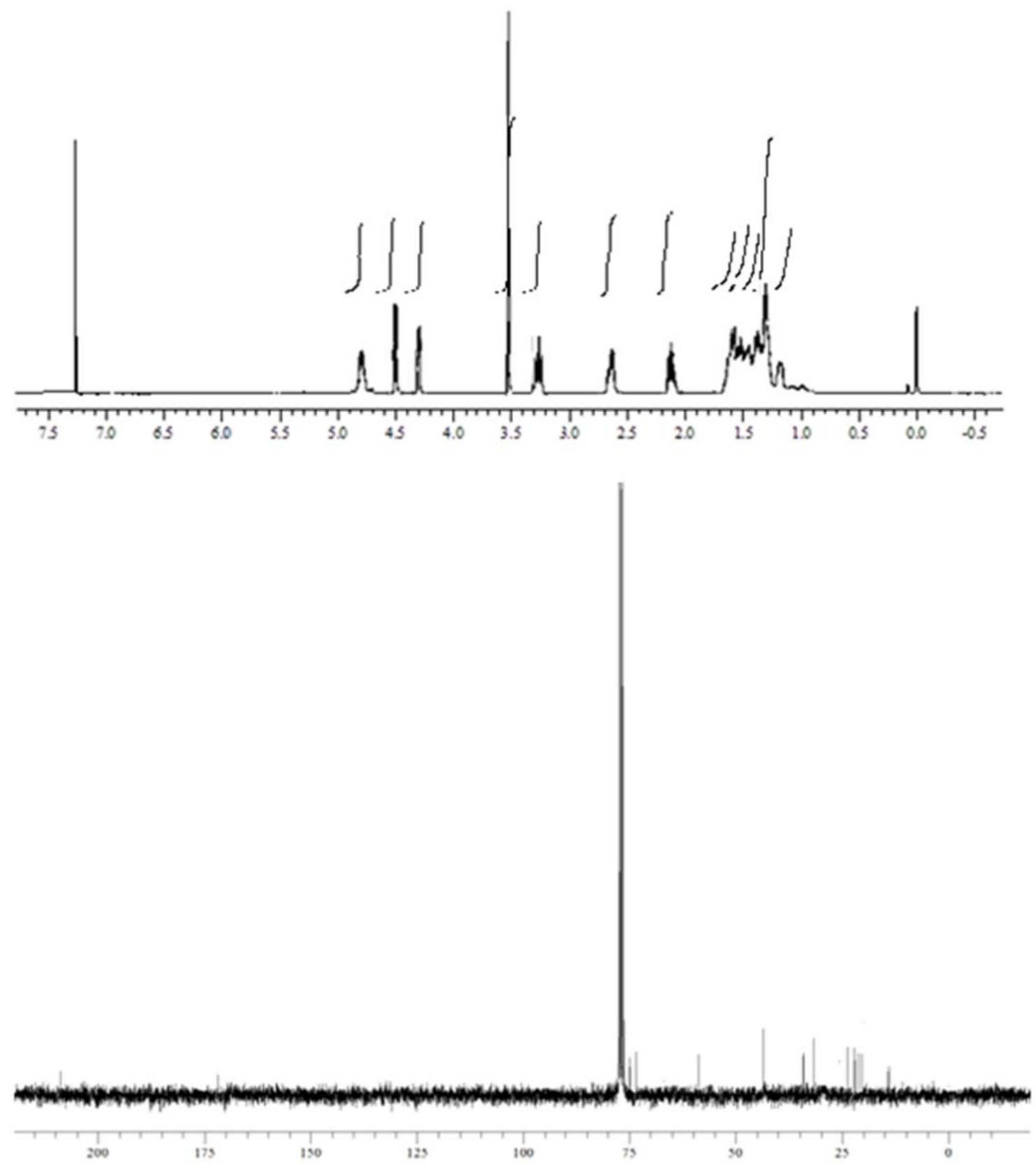

Spectrum 18: ${ }^{1} \mathrm{H}$ NMR Spectrum of $1\left(300 \mathrm{MHz}, \mathrm{CDCl}_{3}\right)$.

${ }^{13} \mathrm{C}$ NMR Spectrum of $\mathbf{1}\left(75 \mathrm{MHz}, \mathrm{CDCl}_{3}\right)$. 

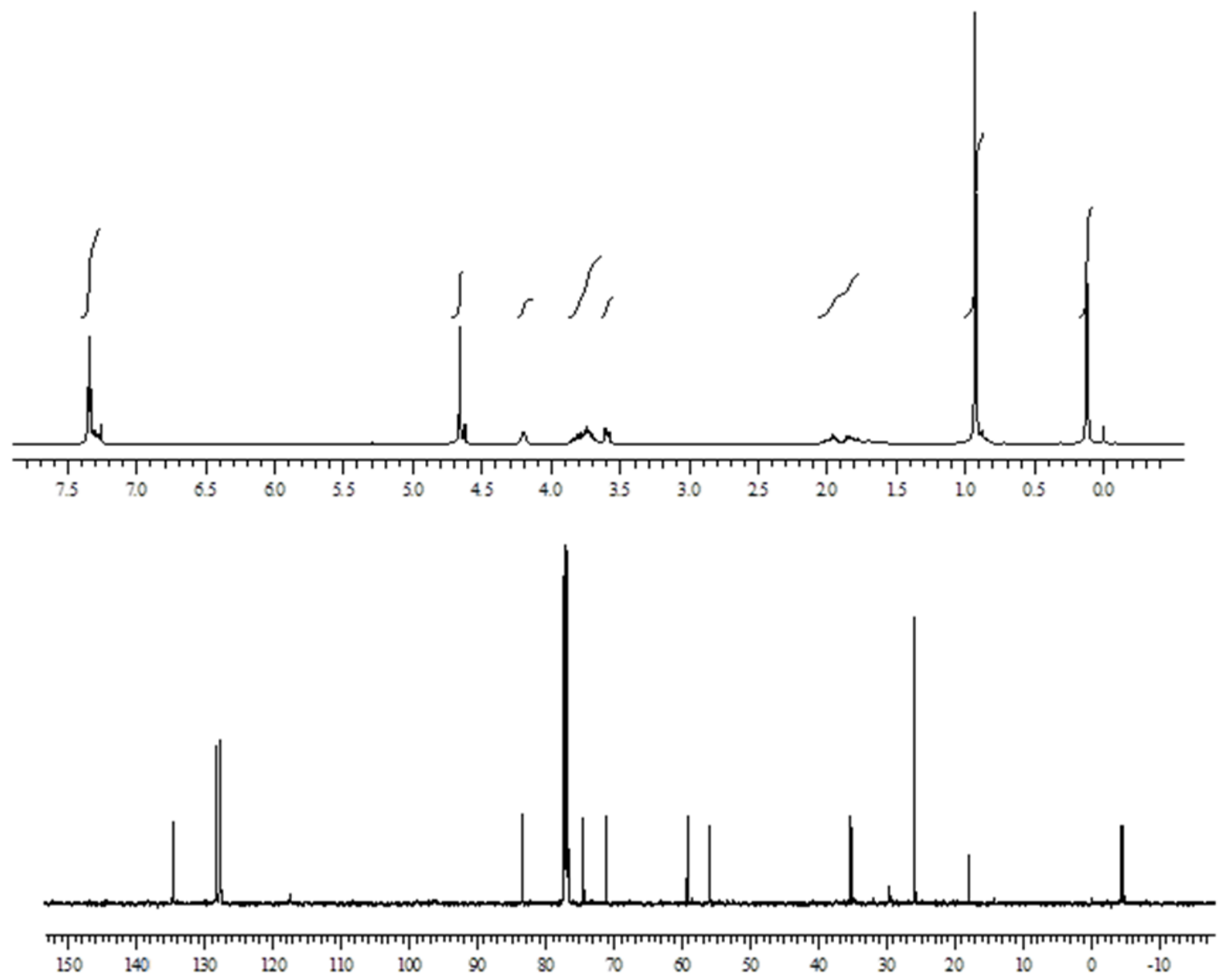

Spectrum 19: ${ }^{1} \mathrm{H}$ NMR Spectrum of 11 a $\left(300 \mathrm{MHz}, \mathrm{CDCl}_{3}\right)$.

${ }^{13} \mathrm{C}$ NMR Spectrum of $11 \mathrm{a}\left(75 \mathrm{MHz}, \mathrm{CDCl}_{3}\right)$. 

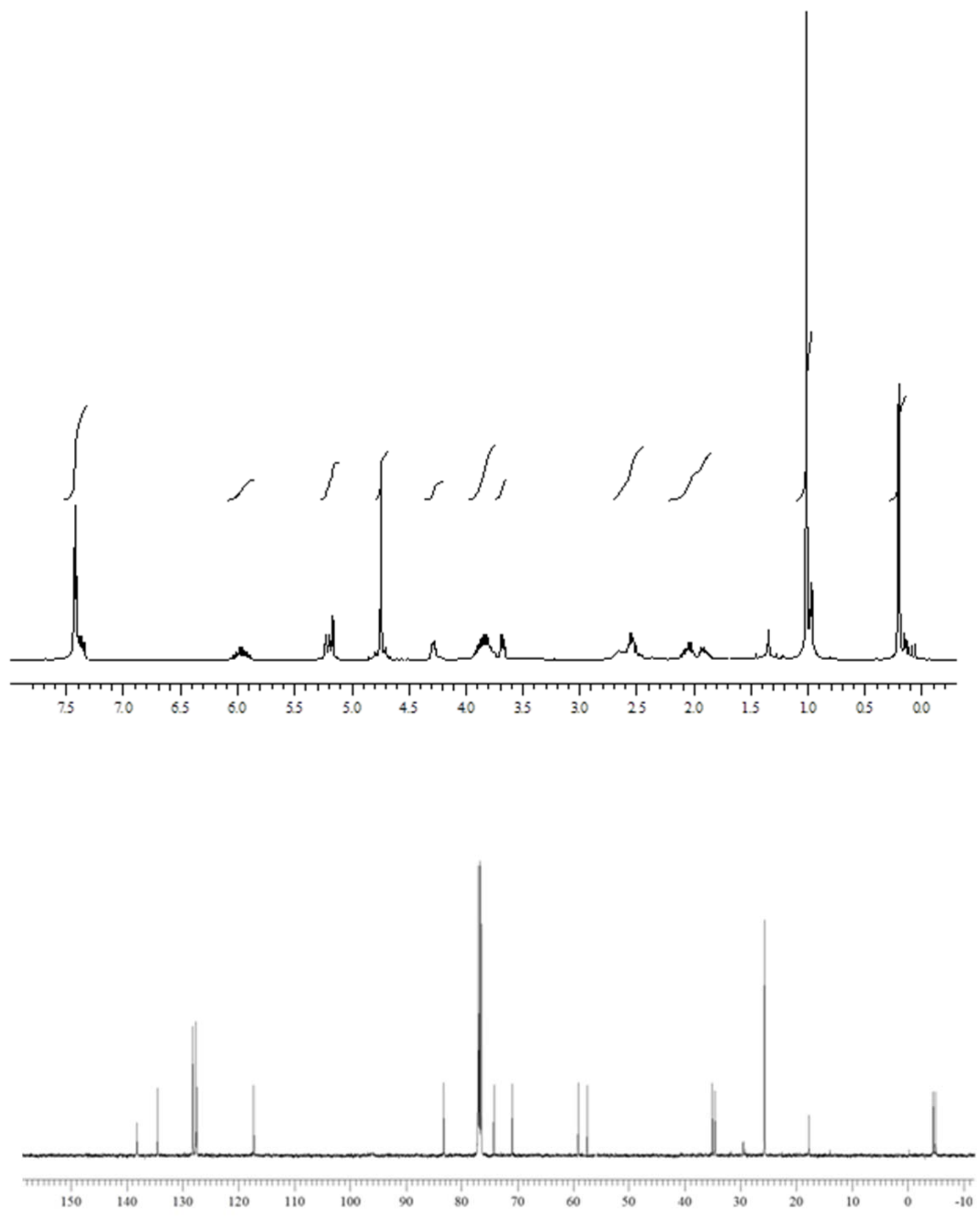

Spectrum 20: ${ }^{1} \mathrm{H}$ NMR Spectrum of $28\left(300 \mathrm{MHz}, \mathrm{CDCl}_{3}\right)$.

${ }^{13} \mathrm{C}$ NMR Spectrum of $28\left(75 \mathrm{MHz}, \mathrm{CDCl}_{3}\right)$. 

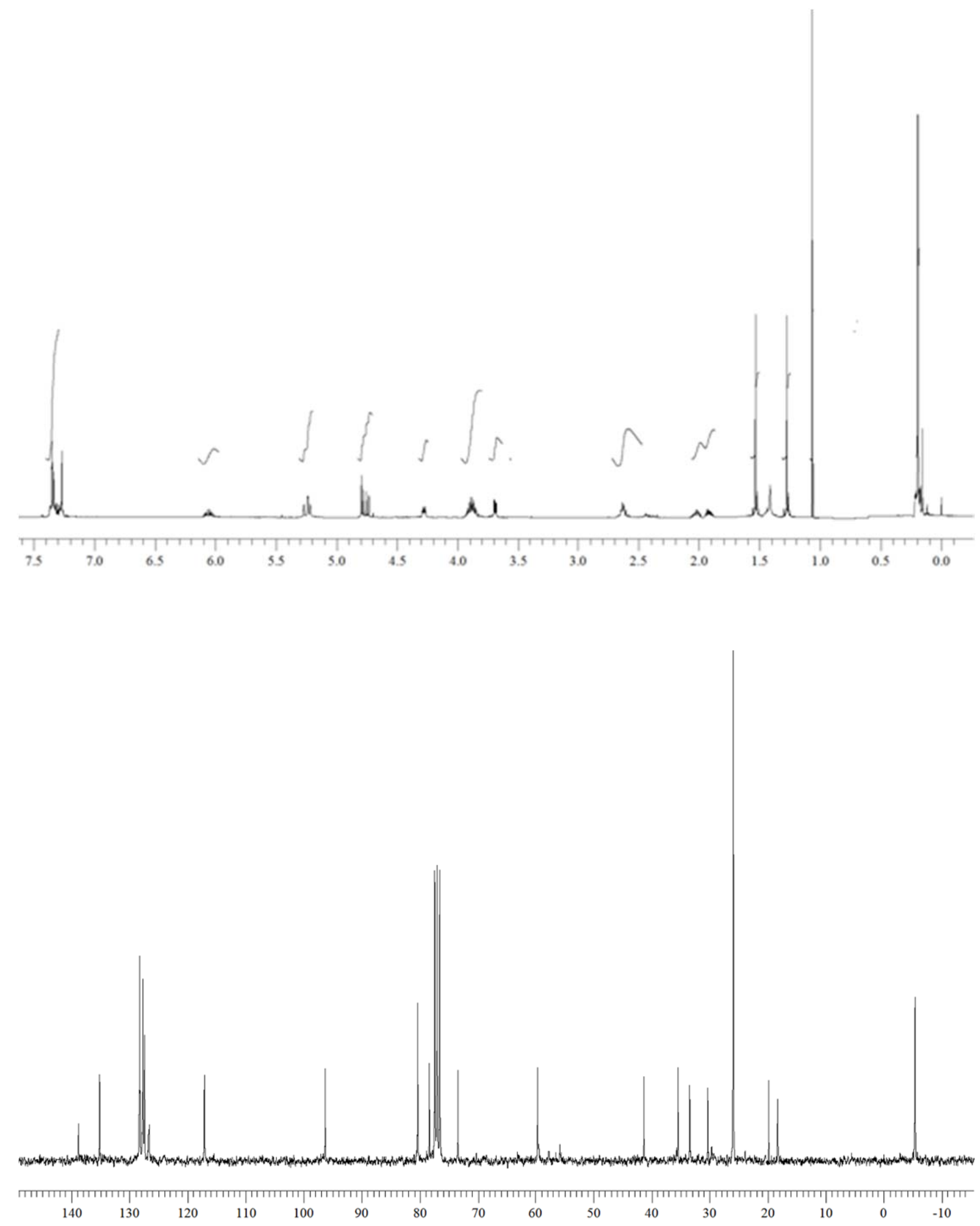

Spectrum 21: ${ }^{1} \mathrm{H}$ NMR Spectrum of $29\left(300 \mathrm{MHz}, \mathrm{CDCl}_{3}\right)$.

${ }^{13} \mathrm{C}$ NMR Spectrum of $29\left(75 \mathrm{MHz}, \mathrm{CDCl}_{3}\right)$. 

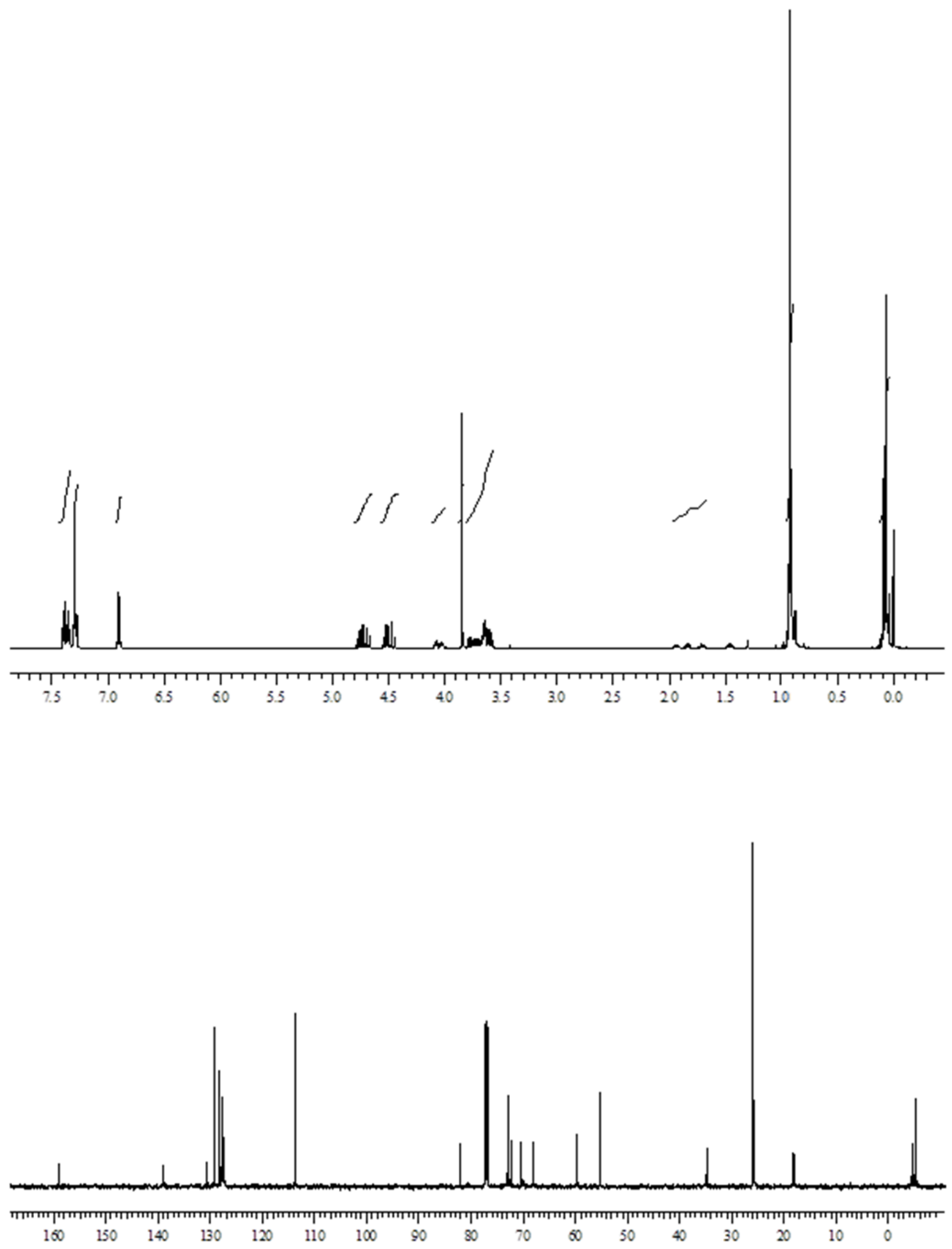

Spectrum 22: ${ }^{1} \mathrm{H}$ NMR Spectrum of $10\left(300 \mathrm{MHz}, \mathrm{CDCl}_{3}\right)$.

${ }^{13} \mathrm{C}$ NMR Spectrum of $\mathbf{1 0}\left(75 \mathrm{MHz}, \mathrm{CDCl}_{3}\right)$. 


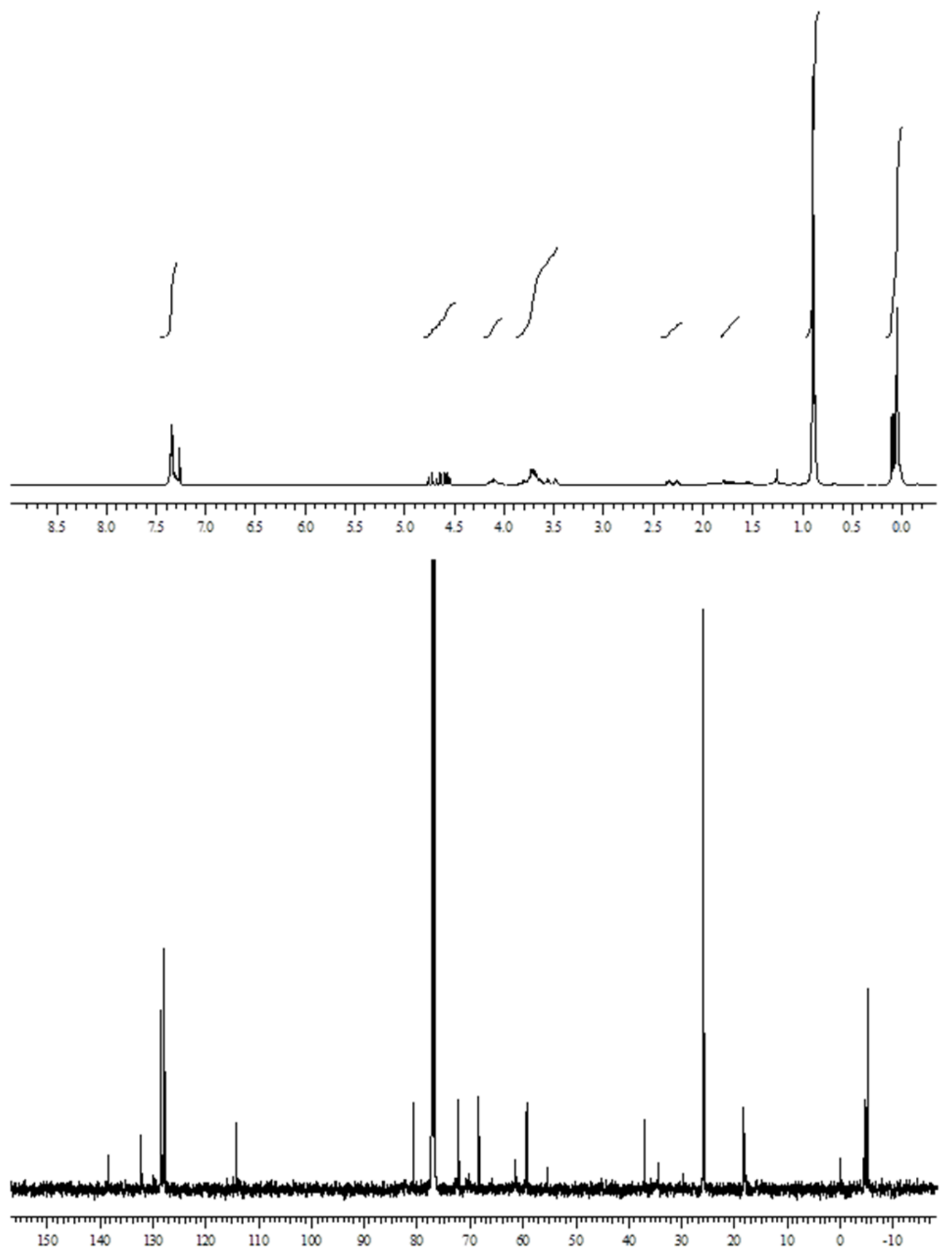

Spectrum 23: ${ }^{1} \mathrm{H}$ NMR Spectrum of $\mathbf{3 0}\left(300 \mathrm{MHz}, \mathrm{CDCl}_{3}\right)$.

${ }^{13} \mathrm{C}$ NMR Spectrum of $\mathbf{3 0}\left(75 \mathrm{MHz}, \mathrm{CDCl}_{3}\right)$. 

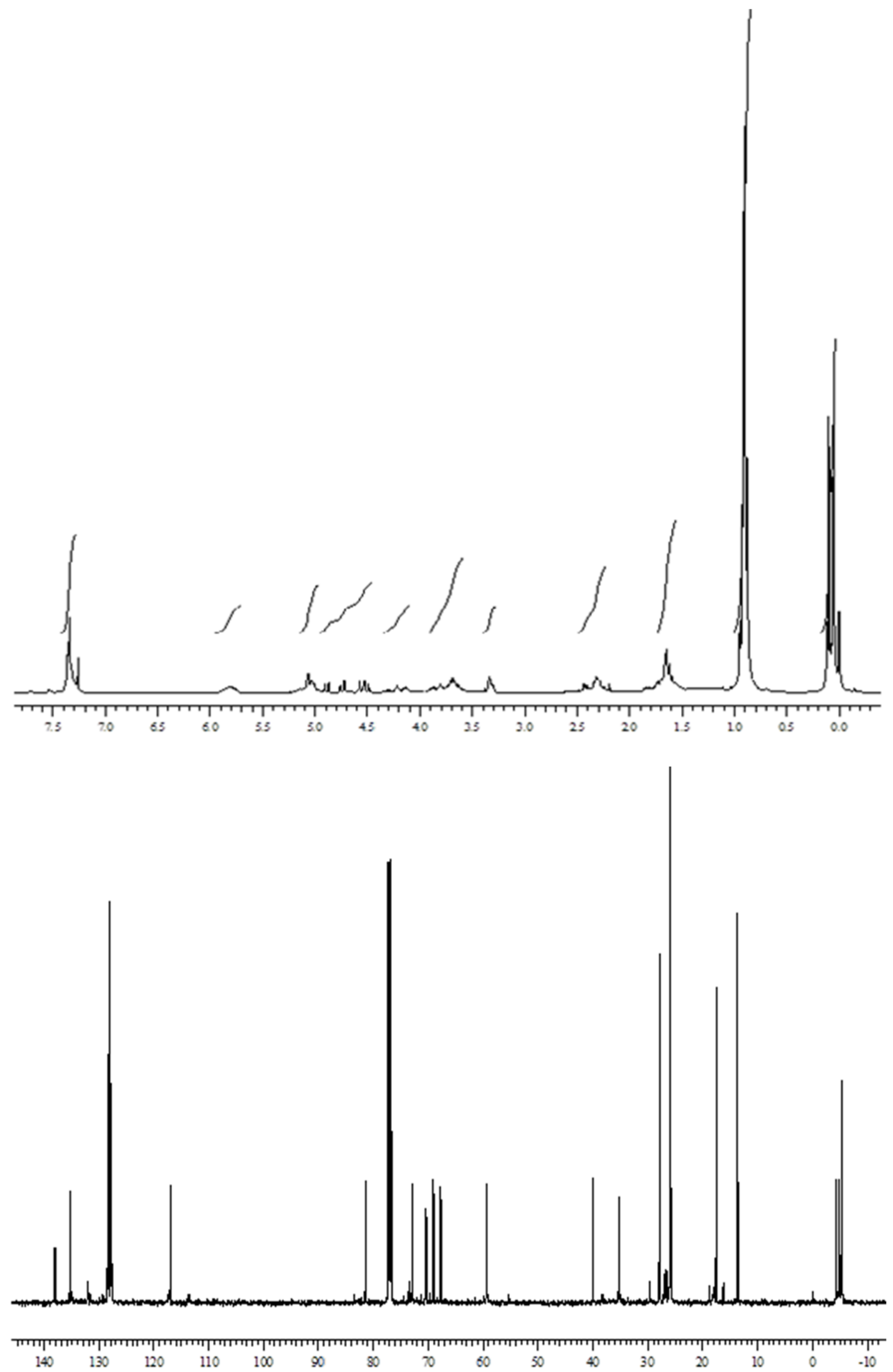

Spectrum 24: ${ }^{1} \mathrm{H}$ NMR Spectrum of $\mathbf{3 1}(300 \mathrm{MHz}, \mathrm{CDCl})$

${ }^{13} \mathrm{C}$ NMR Spectrum of $\mathbf{3 1}(75 \mathrm{MHz}, \mathrm{CDCl} 3)$ 

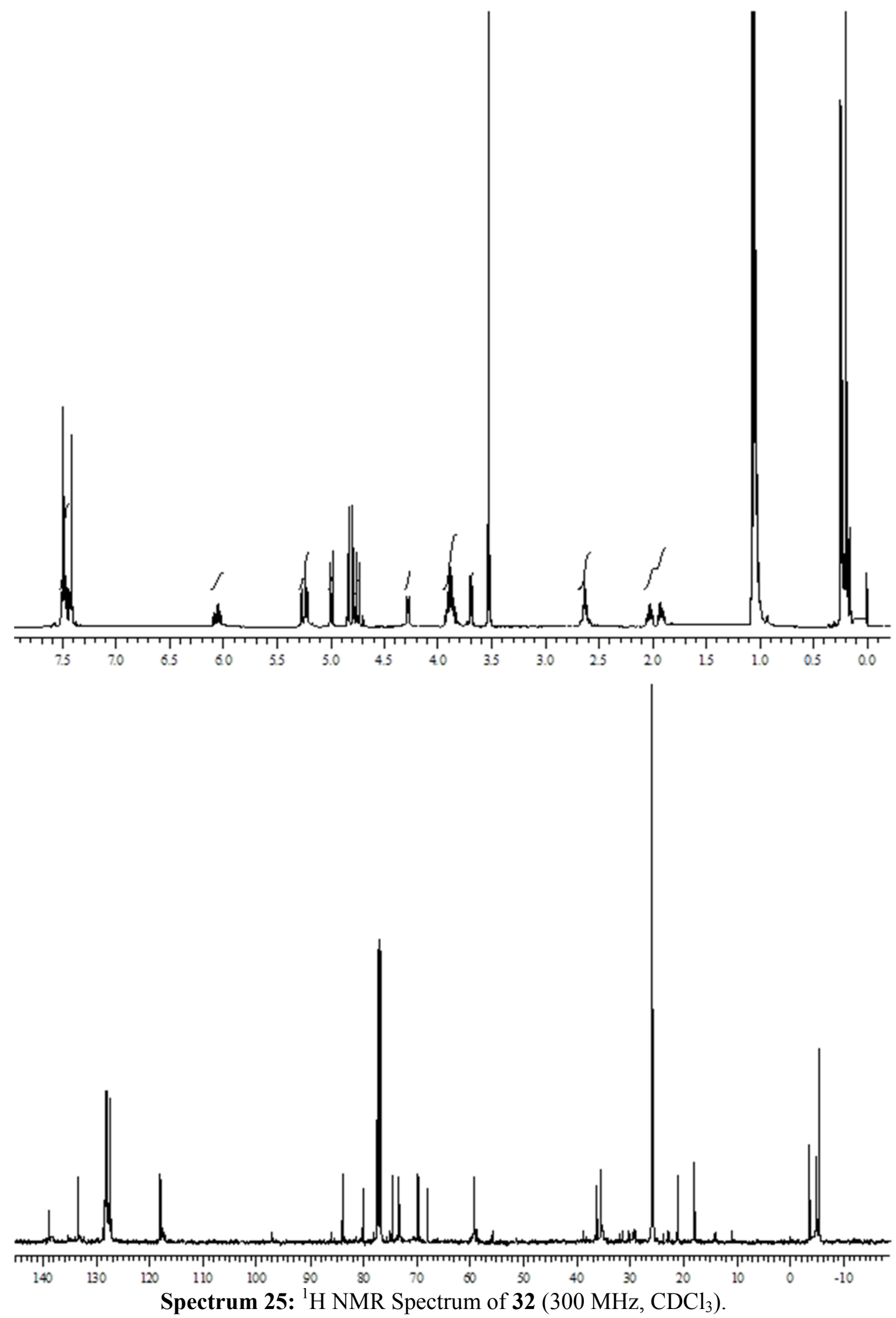

${ }^{13} \mathrm{C}$ NMR Spectrum of $32\left(75 \mathrm{MHz}, \mathrm{CDCl}_{3}\right)$. 


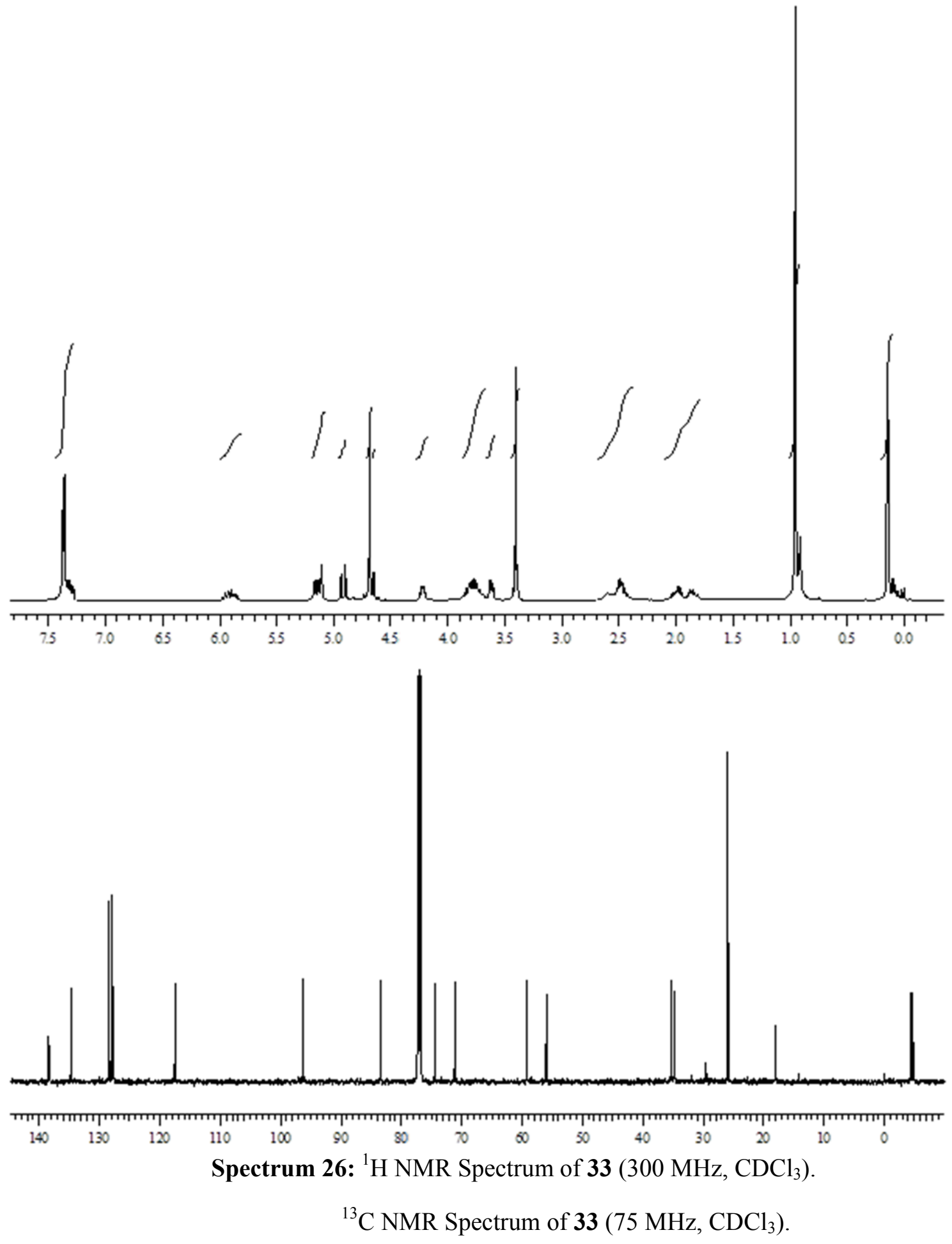




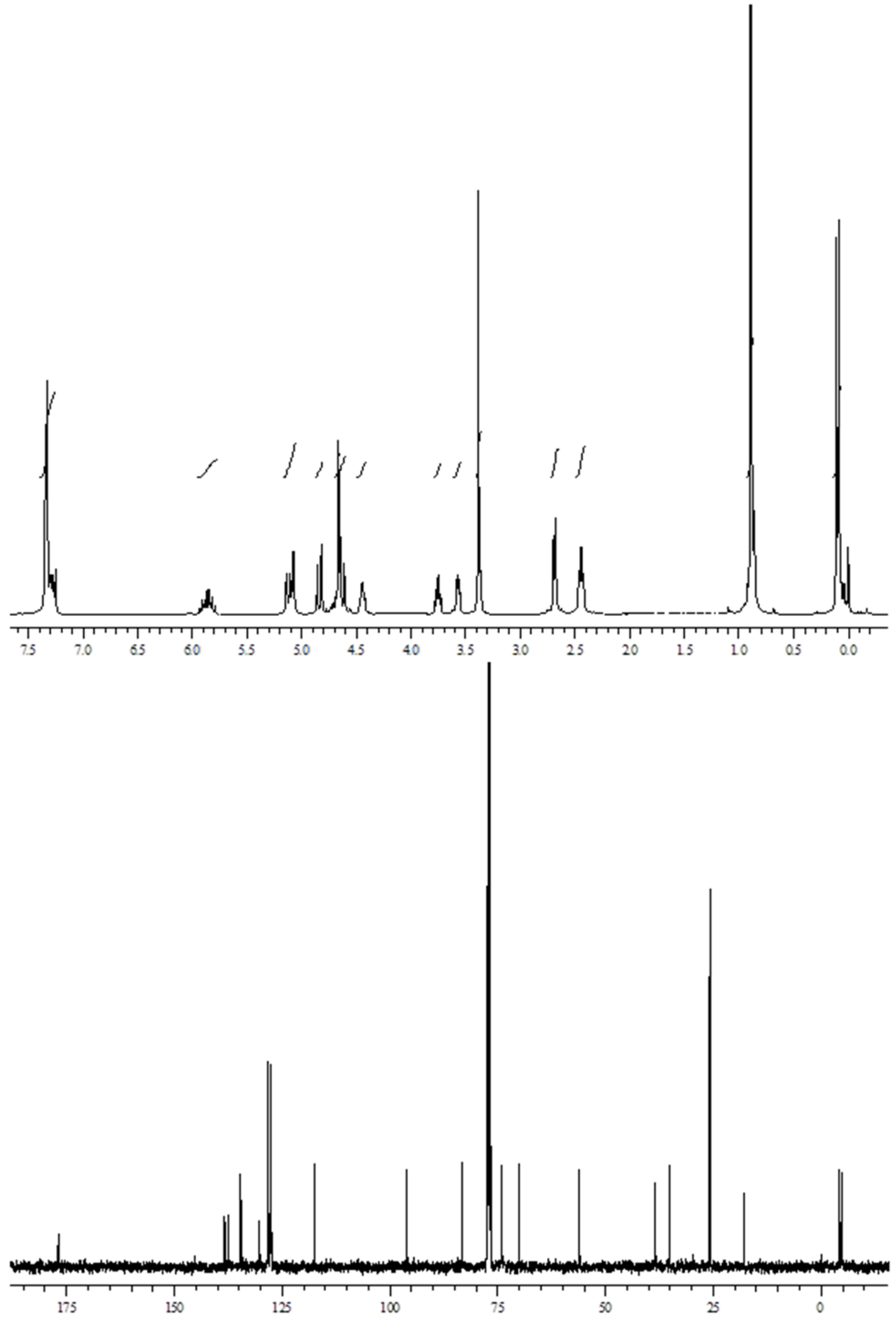

Spectrum 27: ${ }^{1} \mathrm{H}$ NMR Spectrum of 7 (300 MHz, $\left.\mathrm{CDCl}_{3}\right)$.

${ }^{13} \mathrm{C}$ NMR Spectrum of $7\left(75 \mathrm{MHz}, \mathrm{CDCl}_{3}\right)$. 

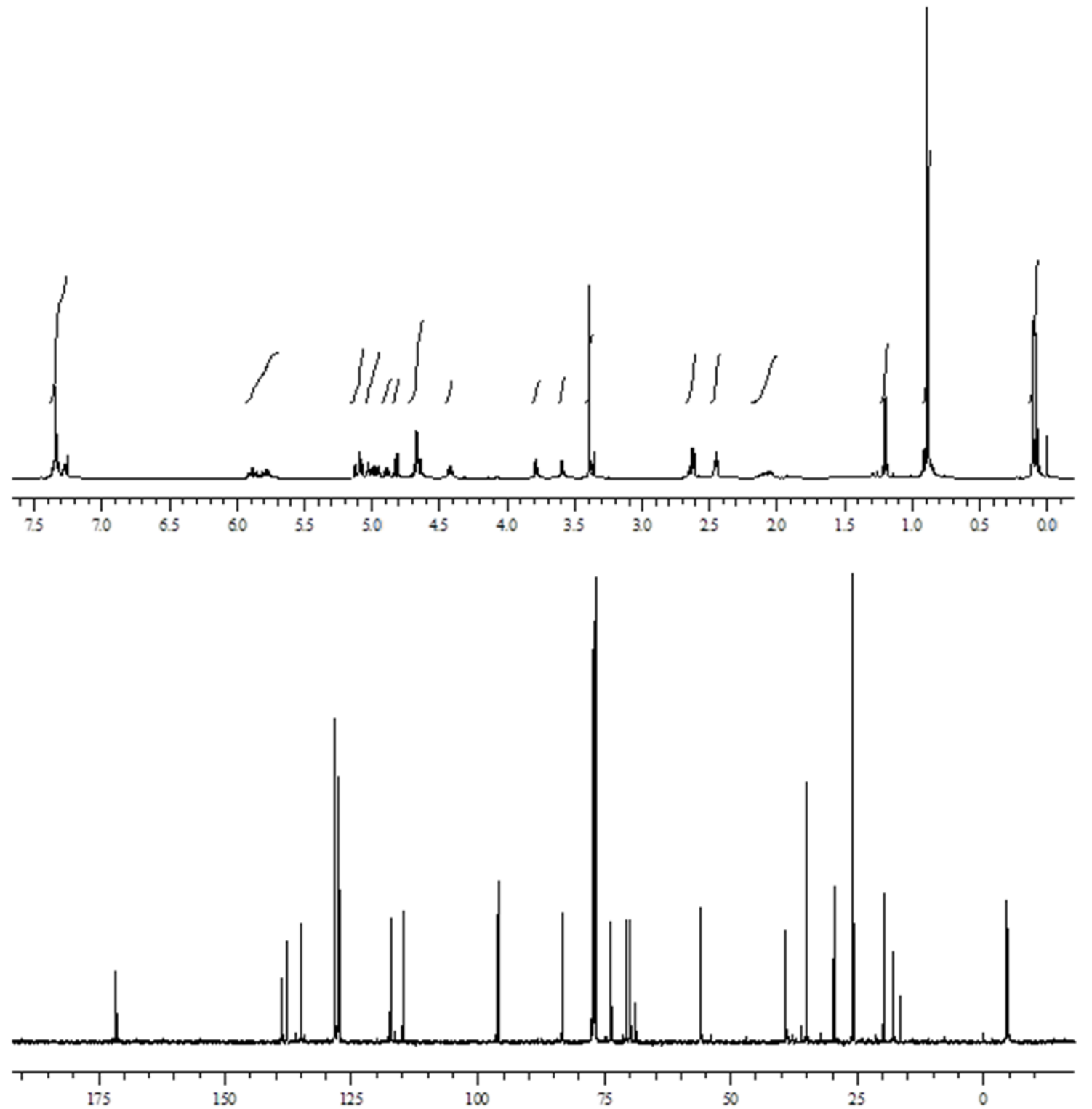

Spectrum 28: ${ }^{1} \mathrm{H}$ NMR Spectrum of 4 (300 MHz, $\left.\mathrm{CDCl}_{3}\right)$.

${ }^{13} \mathrm{C}$ NMR Spectrum of 4 (75 MHz, $\left.\mathrm{CDCl}_{3}\right)$. 